Pacific Journal of Mathematics

EISENSTEIN-SERIES ON REAL, COMPLEX, AND 


\title{
EISENSTEIN-SERIES ON REAL, COMPLEX, AND QUATERNIONIC HALF-SPACES
}

\section{ALOYS KRIEG}

\begin{abstract}
The real, complex, and quaternionic half-spaces are introduced in certain analogy with the Siegel half-space. The modified symplectic group acts on the attached half-space in the usual way. At first properties of these half-spaces considered as symmetric spaces are derived. Then a fundamental domain with respect to the modified modular group, which consists of integral modified symplectic matrices, is constructed. The behavior of convergence of the corresponding Eisenstein-series is determined carefully. The Fourier-coefficients of the Eisenstein-series are calculated explicitly, whenever the degree is sufficiently small.
\end{abstract}

Introduction. The present paper deals with half-spaces, which are built in analogy with the Siegel half-space, and the corresponding nonanalytic Eisenstein-series. The roots can be traced back to C. L. Siegel's paper "Die Modulgruppe in einer einfachen involutorischen Algebra" [30]. A special case of these investigations is considered and continued by the examination of the Riemannian geometry as well as the attached Eisenstein-series.

To be more precise, throughout this paper let $\mathbf{F}$ stand for $\mathbf{R}, \mathbf{C}$ or $\mathbf{H}$, where $\mathbf{H}$ is the skew-field of real Hamiltonian quaternions. Just as in [16] let $r=r(F)=\operatorname{dim}_{\mathbf{R}} \mathbf{F}$ and denote the standard basis of $\mathbf{F}$ over $\mathbf{R}$ by $1=e_{1}, \ldots, e_{r}$. Given $a=\sum_{j=1}^{r} a_{j} e_{j} \in \mathbf{F}, a_{j} \in \mathbf{R}$, put $\operatorname{Re}(a):=a_{1}$ and let $a \mapsto \bar{a}=2 \operatorname{Re}(a)-a$ denote the canonical conjugation in $\mathbf{F}$. Then $A^{(n)}$, resp. $A \in \operatorname{Mat}(n ; \mathbf{F})$, means that $A$ is an $n \times n$ matrix with entries in $\mathbf{F}$ and $A^{\prime}$ denotes the transpose of $A$. The letter $I$ is reserved for the identity matrix and 0 for the zero matrix of appropriate size. $\mathrm{GL}(n ; \mathbf{F})$ stands for the group of units in the $\operatorname{ring} \operatorname{Mat}(n ; \mathbf{F})$.

The half-space $\mathscr{H}(n ; \mathbf{F})$ consists of all $Z \in \operatorname{Mat}(n ; \mathbf{F})$ such that $Z+\bar{Z}^{\prime}$ becomes a positive definite Hermitian matrix. Thus $i \mathscr{H}(n ; \mathbf{C})$ equals the Hermitian half-space, which was investigated by $\mathrm{H}$. Braun [3]. But the remaining cases are related, because $\mathscr{H}(n ; \mathbf{H})$ can always be embedded into the Hermitian half-space of degree $2 n$.

The attached modified symplectic group $\operatorname{MSp}(n ; \mathbf{F})$ consists of the

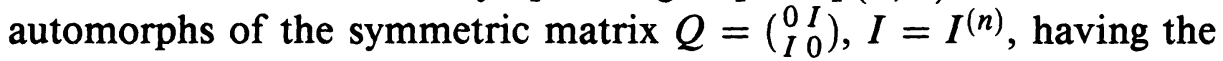


signature $(n, n)$ and acts on $\mathscr{H}(n ; \mathbf{F})$ in the usual way. The real modified symplectic group was already investigated by C. L. Siegel [28], M. Koecher [14], III, $\S 1$, and H. Maaß [23] in different contexts. Considering the symplectic group

$$
\begin{aligned}
\operatorname{Sp}(n ; \mathbf{F}) & =\left\{M \in \operatorname{Mat}(2 n ; \mathbf{F}) ; \bar{M}^{\prime} J M=J\right\}, \\
J & =\left(\begin{array}{cc}
0 & I \\
-I & 0
\end{array}\right), \quad I=I^{(n)},
\end{aligned}
$$

as in [16], one has

$$
\left(\begin{array}{cc}
e_{2} I & 0 \\
0 & I
\end{array}\right) \operatorname{MSp}(n ; \mathbf{C})\left(\begin{array}{cc}
e_{2} I & 0 \\
0 & I
\end{array}\right)^{-1}=\operatorname{Sp}(n ; \mathbf{C}) .
$$

$\operatorname{MSp}(n ; \mathbf{F})$ is obviously conjugate to the indefinite unitary group $\mathrm{U}^{n}(2 n, \mathbf{F})$ in [34], p. 377 , and to $\mathrm{O}(n, n), \mathrm{U}(n, n)$, resp. $\mathrm{Sp}(n, n)$, if $\mathbf{F}=\mathbf{R}, \mathbf{C}$, resp. $\mathbf{H}$, in Helgason's notation (cf. [8], p. 340).

Nevertheless the notion of modified symplectic group may be justified by the connection with C. L. Siegel's paper [30]. Consider $\mathbf{F}=\mathbf{R}, \mathbf{H}$ and an arbitrary $\mathbf{R}$-involution $l$ of $\mathbf{M a t}(n ; \mathbf{F})$. According to [1], $\mathrm{X}$, Theorem 11 , there exists $F \in \mathrm{GL}(n ; \mathbf{F})$ such that $\bar{F}^{\prime}= \pm F$ and

$$
l(X)=F \bar{X}^{\prime} F^{-1} \quad \text { for } X \in \operatorname{Mat}(n ; \mathbf{F}) .
$$

In this general situation C. L. Siegel [30] defined the symplectic group $\Sigma$. In our notation we gain

$$
\Sigma= \begin{cases}\left(\begin{array}{cc}
F & 0 \\
0 & I
\end{array}\right) \operatorname{Sp}(n ; \mathbf{F})\left(\begin{array}{cc}
F & 0 \\
0 & I
\end{array}\right)^{-1} & \text { if } \bar{F}^{\prime}=F, \\
\left(\begin{array}{cc}
F & 0 \\
0 & I
\end{array}\right) \operatorname{MSp}(n ; \mathbf{F})\left(\begin{array}{cc}
F & 0 \\
0 & I
\end{array}\right)^{-1} & \text { if } \bar{F}^{\prime}=-F .\end{cases}
$$

The special case $\mathbf{F}=\mathbf{H}, n=1, F=\left(e_{3}\right)$ was recently treated by E. Kähler [10].

The Riemannian geometry and the description of the geodesics can be pointed out along the lines of Siegel's classical work [29], where the case $\mathbf{F}=\mathbf{C}$ is due to $\mathrm{H}$. Klingen [12]. If $d Z$ denotes the matrix of differentials, then

$$
d s^{2}=\frac{1}{2} \operatorname{trace}\left(Y^{-1} d Z Y^{-1} \overline{d Z^{\prime}}+d Z Y^{-1} \overline{d Z^{\prime}} Y^{-1}\right), \quad Y:=\frac{1}{2}\left(Z+\bar{Z}^{\prime}\right),
$$

proves to be a positive definite quadratic differential form. The modified symplectic transformations become isometries. Thus $\mathscr{H}(n ; \mathbf{F})$ endowed with $d s^{2}$ turns out to be a Riemannian globally symmetric space of the noncompact type, which is irreducible except for 
$\mathbf{F}=\mathbf{R}, n=1,2$ and which fails to be Hermitian, whenever $\mathbf{F}=\mathbf{R}, n \neq$ 2 , resp. $\mathbf{F}=\mathbf{H}, n \geq 1$.

$\mathscr{H}(1 ; \mathbf{C})$ equals the right half-plane in $\mathbf{C}$. Moreover $\mathscr{H}(1 ; \mathbf{H})$ becomes a model of the four-dimensional hyperbolic space, which was recently treated by E. Kähler [10]. Kähler's paper was the starting point of these investigations. The present paper arose from the attempt of combining Kähler's approach with the investigations of Eisenstein-series on the three-dimensional hyperbolic space by $J$. Elstrodt, F. Grunewald and J. Mennicke [6] as well as with Siegel's methods. Therefore this paper can also be understood as an extension of [6].

Choosing a special order for $\mathbf{F}=\mathbf{R}, \mathbf{C}, \mathbf{H}$, namely $\mathbf{Z}$, the Gaussian integers and the quaternions of Hurwitz, the modified modular group is defined to consist of all integral modified symplectic matrices. By means of the Euclidean algorithm a simple set of generators of the modified modular group can be determined. Following the classical procedure as in the case of the Siegel half-space, a fundamental domain is obtained, which has a cusp only at infinity.

The last two paragraphs deal with the corresponding non-analytic Eisenstein-series. Let $\Gamma_{n}$ denote the modified modular group and $\Gamma_{n}^{\infty}$ the subgroup of all matrices, whose $C$-block equals 0 . Given $Z \in$ $\mathscr{H}(n ; \mathbf{F})$ and $M \in \Gamma_{n}$ set $Y_{M}=\frac{1}{2}\left(M\langle Z\rangle+\overline{M\langle Z\rangle}^{\prime}\right)$. Then the Eisensteinseries is given by

$$
E_{n}^{\mathbf{F}}(Z, s)=\sum_{M: \Gamma_{n}^{\infty} \backslash \Gamma_{n}}\left(\operatorname{det} Y_{M}\right)^{s}, \quad Z \in \mathscr{H}(n ; \mathbf{F}),
$$

and converges locally uniformly in $Z$ and $s$. The abscissa of absolute convergence equals $\operatorname{Re}(s)=\frac{1}{n} \cdot d$, where $d$ denotes the dimension of the real vector space of all skew-Hermitian matrices. One can define a modified Siegel $\phi$-operator and obtains the same result, namely

$$
\left.E_{n}^{\mathbf{F}}(\cdot, s)\right|_{s} \phi=E_{n-1}^{\mathbf{F}}(\cdot, s),
$$

as known from the classical case.

The investigations of $E_{n}^{\mathbf{R}}(\cdot, s)$ by $H$. Maaß [23] are extended and partially strengthened. The Eisenstein-series $E_{n}^{\mathbf{C}}(\cdot, s)$ were also examined by G. Shimura [27]. But one has to distinguish carefully between $E_{n}^{\mathbf{H}}(\cdot, s)$ and the analytic Eisenstein-series on the half-space of quaternions in [16], since the domains of definition are completely different. 
Moreover coincidences between different classes of symmetric spaces for "small" values of $n$ (cf. [8], p. 351-353) correspond to identities between the associated Eisenstein-series. Therefore Eisensteinseries on the upper half-plane in $\mathbf{C}$ as well as Eisenstein-series for $\mathrm{GL}(4 ; \mathrm{Z})$ (cf. [31]) come to light.

Finally the Fourier-expansions of Eisenstein-series are investigated. Just as in the case of the Siegel half-space, one cannot expect explicit formulas for arbitrary degree. But if the degree is sufficiently "small", the explicit description of the Fourier-coefficients succeeds. As one can expect from the upper half-plane (cf. [19], [20]), resp. the threedimensional hyperbolic space (cf. [6]), resp. from Eisenstein-series for $\mathrm{GL}(n ; \mathbf{Z})$ (cf. [31]), the Fourier-coefficients involve the modified Bessel function and certain weighted divisor sums.

Although a great deal of work can be done along the lines of classical patterns, one has to be cautious with the analogy. On several occasions the cases $\mathbf{F}=\mathbf{R}$ or $\mathbf{F}=\mathbf{H}$ or even $n=1$ have to be treated in a different way. Thus an explicit description might be useful.

The author would like to express his gratitude to Professor Dr. M. Koecher for his encouragement and helpful advice, as well as to Professor Dr. J. Elstrodt and Professor S. Raghavan for their suggestions.

1. Real, complex, and quaternionic half-space. Considering the symmetric matrix

$$
Q:=\left(\begin{array}{cc}
0 & I \\
I & 0
\end{array}\right), \quad I=I^{(n)}
$$

we define

$$
\operatorname{MSp}(n ; \mathbf{F}):=\left\{M \in \operatorname{Mat}(2 n ; \mathbf{F}) ; \bar{M}^{\prime} Q M=Q\right\}
$$

and call $\operatorname{MSp}(n ; \mathbf{F})$ the modified symplectic group of degree $n$ over F. Given $M=\left(\begin{array}{ll}A & B \\ C & D\end{array}\right) \in \operatorname{MSp}(n ; \mathbf{F})$ we always assume $A, B, C, D \in$ $\operatorname{Mat}(n ; \mathbf{F})$. Clearly $M \in \operatorname{MSp}(n ; \mathbf{F})$ is equivalent to $\bar{M}^{\prime} \in \operatorname{MSp}(n ; \mathbf{F})$ as well as to

$$
A \bar{B}^{\prime}+B \bar{A}^{\prime}=C \bar{D}^{\prime}+D \bar{C}^{\prime}=0, \quad A \bar{D}^{\prime}+B \bar{C}^{\prime}=I .
$$

In this case one has

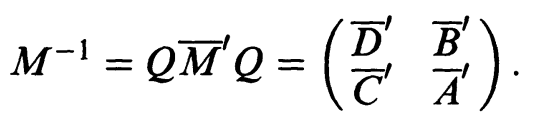


The definition contains one trivial case, namely

$$
\begin{aligned}
\operatorname{MSp}(1 ; \mathbf{R})= & \left\{\left(\begin{array}{ll}
a & 0 \\
0 & a^{-1}
\end{array}\right) ; 0 \neq a \in \mathbf{R}\right\} \\
& \cup\left\{\left(\begin{array}{ll}
0 & b \\
b^{-1} & 0
\end{array}\right) ; 0 \neq b \in \mathbf{R}\right\} .
\end{aligned}
$$

Again in the general situation we want to describe special elements. Therefore we need the real vector space

$$
\operatorname{Alt}(n ; \mathbf{F}):=\left\{X \in \operatorname{Mat}(n ; \mathbf{F}) ; \bar{X}^{\prime}=-X\right\}
$$

of all skew-Hermitian matrices, which has the dimension $\frac{1}{2} r n(n+1)-$ $n$. Then the matrices

$$
\begin{gathered}
Q=\left(\begin{array}{ll}
0 & I \\
I & 0
\end{array}\right), \quad\left(\begin{array}{ll}
I & S \\
0 & I
\end{array}\right), \quad S \in \operatorname{Alt}(n ; \mathbf{F}), \\
\left(\begin{array}{ll}
\bar{U}^{\prime} & 0 \\
0 & U^{-1}
\end{array}\right), \quad U \in \mathrm{GL}(n ; \mathbf{F}),
\end{gathered}
$$

belong to $\operatorname{MSp}(n ; \mathbf{F})$ in view of $(1.1)$.

Moreover consider the subgroup

$$
\operatorname{MSp}(n ; \mathbf{F})_{\infty}:=\left\{M=\left(\begin{array}{cc}
A & B \\
C & D
\end{array}\right) \in \operatorname{MSp}(n ; \mathbf{F}) ; C=0\right\} .
$$

Then (1.1) immediately yields

$$
\begin{aligned}
\operatorname{MSp}(n ; \mathbf{F})_{\infty}=\left\{\left(\begin{array}{ll}
\bar{U}^{\prime} & 0 \\
0 & U^{-1}
\end{array}\right)\right. & \left(\begin{array}{ll}
I & S \\
O & I
\end{array}\right) \\
U & \in \operatorname{GL}(n ; \mathbf{F}), S \in \operatorname{Alt}(n ; \mathbf{F})\} .
\end{aligned}
$$

Given $0<j<n$ we define the usual embedding

$$
\operatorname{MSp}(j ; \mathbf{F}) \times \operatorname{MSp}(n-j ; \mathbf{F}) \rightarrow \operatorname{MSp}(n ; \mathbf{F}), \quad\left(M_{1}, M_{2}\right) \mapsto M_{1} \times M_{2},
$$

$$
\left(\begin{array}{ll}
A_{1} & B_{1} \\
C_{1} & D_{1}
\end{array}\right) \times\left(\begin{array}{ll}
A_{2} & B_{2} \\
C_{2} & D_{2}
\end{array}\right):=\left(\begin{array}{llll}
A_{1} & 0 & B_{1} & 0 \\
0 & A_{2} & 0 & B_{2} \\
C_{1} & 0 & D_{1} & 0 \\
0 & C_{2} & 0 & D_{2}
\end{array}\right)
$$

(cf. [16], p. 44). If $M=\left(\begin{array}{ll}A & B \\ C & D\end{array}\right) \in \operatorname{MSp}(n ; \mathbf{F})$ with rank $C=j$, one can proceed as in the classical situation (cf. [4], 3.12, [16], II.1.4) in order to obtain $K, L \in \operatorname{MSp}(n ; \mathbf{F})_{\infty}$ such that

$$
M=K\left(Q^{(2 j)} \times I\right) L,
$$

where $j=0, n$ can be interpreted unmistakably. 
LEMMA 1.1. (a) The group $\operatorname{MSp}(n ; \mathbf{F})$ is generated by the matrices

$$
\begin{gathered}
Q^{(2)} \times I, \quad\left(\begin{array}{ll}
I & S \\
0 & I
\end{array}\right), \quad S \in \operatorname{Alt}(n ; \mathbf{F}), \\
\left(\begin{array}{ll}
\bar{U}^{\prime} & 0 \\
0 & U^{-1}
\end{array}\right), \quad U \in \operatorname{GL}(n ; \mathbf{F}) .
\end{gathered}
$$

(b) Let $\mathbf{F}=\mathbf{R}, n$ odd, or $\mathbf{F}=\mathbf{C}, \mathbf{H}, n \geq 1$. Then $\operatorname{MSp}(n ; \mathbf{F})$ is also generated by the matrices (1.4).

Proof. (a) Apply (1.7).

(b) If $\mathbf{F}=\mathbf{C}, \mathbf{H}$, compute

$$
Q^{(2)} \times I=\left(\left(\begin{array}{cc}
I & S \\
0 & I
\end{array}\right)\left(\begin{array}{ll}
0 & I \\
I & 0
\end{array}\right)\right)^{2}\left(\begin{array}{ll}
I & S \\
0 & I
\end{array}\right)\left(\begin{array}{ll}
\bar{U}^{\prime} & 0 \\
0 & U^{-1}
\end{array}\right),
$$

where $S=\left(\begin{array}{cc}e_{2} & 0 \\ 0 & 0\end{array}\right) \in \operatorname{Alt}(n ; \mathbf{F}), U=\left(\begin{array}{cc}e_{2} & 0 \\ 0 & I\end{array}\right) \in \mathrm{GL}(n ; \mathbf{F})$. If $\mathbf{F}=\mathbf{R}, n=1$ use (1.3). In the case $\mathbf{F}=\mathbf{R}, n=2 m+1, m \geq 1$, compute

$$
Q^{(2)} \times I=\left(\left(\begin{array}{ll}
I & S \\
0 & I
\end{array}\right)\left(\begin{array}{ll}
0 & I \\
I & 0
\end{array}\right)\right)^{3}\left(\begin{array}{ll}
U^{\prime} & 0 \\
0 & U^{-1}
\end{array}\right),
$$

where $S=\left(\begin{array}{cc}0 & 0 \\ 0 & J\end{array}\right) \in \operatorname{Alt}(n ; \mathbf{R}), U=\left(\begin{array}{ll}1 & 0 \\ 0 & J\end{array}\right) \in \mathrm{GL}(n ; \mathbf{R}), J=J^{(2 m)}$.

The case $\mathbf{F}=\mathbf{R}$ has to be treated in a different way. Note that $\operatorname{Sp}(n ; \mathbf{R}) \subset \operatorname{SL}(2 n ; \mathbf{R})$, whereas $(1.5)$ and $(1.7)$ yield the surprising formula

$$
\operatorname{det} M=(-1)^{j}, \quad j=\operatorname{rank} C,
$$

whenever $M=\left(\begin{array}{ll}A & B \\ C & D\end{array}\right) \in \operatorname{MSp}(n ; \mathbf{R})$. Thus $\operatorname{MSp}(n ; \mathbf{R}) \cap \operatorname{SL}(2 n ; \mathbf{R})$ becomes a normal subgroup of $\operatorname{MSp}(n ; \mathbf{R})$ of index 2. If $n$ is even, this subgroup is generated by the matrices (1.4).

Combining (0.2) and (0.3) with Siegel's procedure [30], it becomes obvious how the attached half-space has to be defined. Consider the real vector space

$$
\operatorname{Sym}(n ; \mathbf{F}):=\left\{X \in \operatorname{Mat}(n ; \mathbf{F}) ; \bar{X}^{\prime}=X\right\}
$$

of the dimension $n+\frac{1}{2} r n(n-1)$ as well as the open subset $\operatorname{Pos}(n ; \mathbf{F})$ consisting of all positive definite matrices in $\operatorname{Sym}(n ; \mathbf{F})$. Then set

$$
\begin{aligned}
\mathscr{H}(n ; \mathbf{F}) & =\operatorname{Alt}(n ; \mathbf{F})+\operatorname{Pos}(n ; \mathbf{F}) \\
& =\left\{Z \in \operatorname{Mat}(n ; \mathbf{F}) ; Z+\bar{Z}^{\prime} \in \operatorname{Pos}(n ; \mathbf{F})\right\} .
\end{aligned}
$$

We always assume that each $Z \in \mathscr{H}(n ; \mathbf{F})$ is given in the form

$$
Z=X+Y, \quad X \in \operatorname{Alt}(n ; \mathbf{F}), \quad Y \in \operatorname{Pos}(n ; \mathbf{F}) .
$$


Definition. $\mathscr{H}(n ; \mathbf{F})$ is called the real, complex, resp. quaternionic half-space of degree $n$, whenever $\mathbf{F}=\mathbf{R}, \mathbf{C}$, resp. $\mathbf{H}$.

The definition especially yields

$$
\begin{aligned}
& \mathscr{H}(1 ; \mathbf{R})=\mathbf{R}^{+}=\{y \in \mathbf{R} ; y>0\} \\
& \mathscr{H}(1 ; \mathbf{H})=\left\{z=\sum_{j=1}^{4} z_{j} e_{j} ; z_{j} \in \mathbf{R}, z_{1}>0\right\} .
\end{aligned}
$$

Note that in the cases $\mathbf{F}=\mathbf{R}, \mathbf{H}$ there is a decisive difference between $\mathscr{H}(n ; \mathbf{F})$ and the half-space $H(n ; \mathbf{F})$ defined in [16], p. 46. But there are also close relations, namely

$$
H(n ; \mathbf{C})=i \cdot \mathscr{H}(n ; \mathbf{C})=\operatorname{Sym}(n ; \mathbf{C})+i \operatorname{Pos}(n ; \mathbf{C}) .
$$

Given $a=\sum_{j=1}^{4} a_{j} e_{j} \in \mathbf{H}$ define

$$
\check{a}=\left(\begin{array}{cc}
a_{1} e_{1}+a_{2} e_{2} & a_{3} e_{1}+a_{4} e_{2} \\
-a_{3} e_{1}+a_{4} e_{2} & a_{1} e_{1}-a_{2} e_{2}
\end{array}\right) \in \operatorname{Mat}(2 ; \mathbf{C})
$$

and $\check{A}=\left(\check{a}_{k l}\right) \in \operatorname{Mat}(2 n ; \mathbf{C})$ for $A=\left(a_{k l}\right) \in \operatorname{Mat}(n ; \mathbf{H})$ (cf. [16], p. $14,15,46)$. Then (1.9) leads to

$$
\text { (1.10) } i \check{Z}=i \check{X}+i \check{Y} \in H(2 n ; \mathbf{C}) \text {, whenever } Z=X+Y \in \mathscr{H}(n ; \mathbf{H}) \text {. }
$$

Note that $i$ and $e_{2}$ may be identified for $\mathbf{F}=\mathbf{C}$. Furthermore (0.2) implies

$$
\left(\begin{array}{cc}
i I & 0 \\
0 & I
\end{array}\right)\{\check{M} ; M \in \operatorname{MSp}(n ; \mathbf{H})\}\left(\begin{array}{cc}
i I & 0 \\
0 & I
\end{array}\right)^{-1} \subset \operatorname{Sp}(2 n ; \mathbf{C})
$$

where $I=I^{(2 n)}$. Moreover we have the obvious relations

$$
\begin{gathered}
\mathscr{H}(n ; \mathbf{R}) \subset \mathscr{H}(n ; \mathbf{C}) \subset \mathscr{H}(n ; \mathbf{H}), \\
\operatorname{MSp}(n ; \mathbf{R}) \subset \operatorname{MSp}(n ; \mathbf{C}) \subset \operatorname{MSp}(n ; \mathbf{H}) .
\end{gathered}
$$

We need the abbreviation $A[B]:=\bar{B}^{\prime} A B$, whenever $A$ is an $n \times n$ and $B$ an $n \times m$ matrix, as well as $|\operatorname{det} A|:=|\operatorname{det} \check{A}|^{1 / 2}$, whenever $A \in \operatorname{Mat}(n ; \mathbf{H})$ (cf. [16], p. 15, I.3.4, I.3.5).

Proposition 1.2. The half-space $\mathscr{H}(n ; \mathbf{F})$ is an open convex subset of $\operatorname{Mat}(n ; \mathbf{F})$, which is contained in $\mathrm{GL}(n ; \mathbf{F})$. Given $Z=X+Y \in \mathscr{H}(n ; \mathbf{F})$, one has

$$
|\operatorname{det} Z|^{2}=\operatorname{det} Y \cdot \operatorname{det}\left(Y+Y^{-1}[X]\right) .
$$


Proof.

$$
\begin{aligned}
|\operatorname{det} Z|^{2} & =|\operatorname{det} Z|\left|\operatorname{det} \bar{Z}^{\prime}\right|=\operatorname{det} Y \cdot|\operatorname{det}(X+Y)| \cdot\left|\operatorname{det}\left(-Y^{-1} X+I\right)\right| \\
& =\operatorname{det} Y \cdot \operatorname{det}\left(Y-X Y^{-1} X\right) .
\end{aligned}
$$

The remaining parts are obvious.

Next we consider the action of the modified symplectic group on the attached half-space.

THEOREM 1.3. Let $L, M=\left(\begin{array}{ll}A & B \\ C & D\end{array}\right) \in \operatorname{MSp}(n ; \mathbf{F})$ and $Z=X+Y \in$ $\mathscr{H}(n ; \mathbf{F})$. Then the following hold:

(a) $M\{Z\}:=C Z+D \in \mathrm{GL}(n ; \mathbf{F})$.

(b) $M\langle Z\rangle:=(A Z+B)(C Z+D)^{-1}=X_{M}+Y_{M} \in \mathscr{H}(n ; \mathbf{F})$.

(c) $Y_{M}=Y\left[M\{Z\}^{-1}\right], Y_{M}^{-1}=Y^{-1}\left[\bar{X}^{\prime} \bar{C}^{\prime}+\bar{D}^{\prime}\right]+Y\left[\bar{C}^{\prime}\right]$.

(d) $(L M)\{Z\}=L\{M\langle Z\rangle\} \cdot M\{Z\}$.

The group $\operatorname{MSp}(n ; \mathbf{F})$ acts transitively on $\mathscr{H}(n ; \mathbf{F})$. Two transformations $Z \mapsto M\langle Z\rangle$ and $Z \mapsto L\langle Z\rangle$ coincide if and only if

$$
L=\rho M, \quad \text { where } \rho \in \text { center } \mathbf{F},|\rho|=1 .
$$

Proof. (a) Apply (1.5), (1.7) and Proposition 1.2.

(b), (c) According to (a) we obtain $X_{M} \in \operatorname{Alt}(n ; \mathbf{F}), Y_{M} \in \operatorname{Sym}(n ; \mathbf{F})$ satisfying $M\langle Z\rangle=X_{M}+Y_{M} \in \operatorname{Mat}(n ; \mathbf{F})$. Thus we gain

$$
2 Y_{M}=M\langle Z\rangle+\overline{M\langle Z\rangle}^{\prime}=2 Y\left[(M\{Z\})^{-1}\right]
$$

in view of (1.1). Hence $Y_{M} \in \operatorname{Pos}(n ; \mathbf{F})$ follows. The remaining parts can be derived by easy calculations.

Clearly the definition yields

$$
Z \in \mathscr{H}(n ; \mathbf{F}) \Rightarrow \bar{Z}^{\prime} \in \mathscr{H}(n ; \mathbf{F}) .
$$

In the cases $\mathbf{F}=\mathbf{C}, n \geq 2$, and $\mathbf{F}=\mathbf{H}, n=2$, additionally

$$
Z \in \mathscr{H}(n ; \mathbf{F}) \Rightarrow Z^{\prime} \in \mathscr{H}(n ; \mathbf{F})
$$

holds. Now we are going to describe the combination of (1.13) with the action of $\operatorname{MSp}(n ; \mathbf{F})$ on $\mathscr{H}(n ; \mathbf{F})$. Given $M=\left(\begin{array}{ll}A & B \\ C & D\end{array}\right) \in \operatorname{MSp}(n ; \mathbf{F})$ one easily verifies

$$
\tilde{M}:=M\left[\left(\begin{array}{cc}
I & 0 \\
0 & -I
\end{array}\right)\right]=\left(\begin{array}{cc}
A & -B \\
-C & D
\end{array}\right) \in \operatorname{MSp}(n ; \mathbf{F}) .
$$


Then a calculation using (1.1) and Theorem 1.3 implies has

Proposition 1.4. Given $Z, W \in \mathscr{H}(n ; \mathbf{F})$ and $M \in \operatorname{MSp}(n ; \mathbf{F})$, one

(a) $\overline{M\left\langle\bar{Z}^{\prime}\right.}{ }^{\prime}=\tilde{M}\langle Z\rangle$.

(b) $M\langle Z\rangle+\overline{M\langle W\rangle^{\prime}}=\overline{M\{W\}^{\prime}}-1\left(Z+\bar{W}^{\prime}\right)(M\{Z\})^{-1}$.

(c) $M\langle Z\rangle-M\langle W\rangle={\overline{\tilde{M}\left\{\bar{W}^{\prime}\right.}}^{-1}(Z-W)(M\{Z\})^{-1}$

$$
={\overline{\tilde{M}\left\{\bar{Z}^{\prime}\right.}}^{\prime-1}(Z-W)(M\{W\})^{-1} \text {. }
$$

Following C. L. Siegel [30] we obtain a bijection between the halfspace and the set of positive definite modified symplectic matrices. Put

$$
\mathscr{P}(n ; \mathbf{F}):=\operatorname{MSp}(n ; \mathbf{F}) \cap \operatorname{Pos}(2 n ; \mathbf{F}) .
$$

THEOREM 1.5. The map

$$
\kappa: \mathscr{H}(n ; \mathbf{F}) \rightarrow \mathscr{P}(n ; \mathbf{F}), \quad Z=X+Y \mapsto\left(\begin{array}{ll}
Y^{-1} & 0 \\
0 & Y
\end{array}\right)\left[\left(\begin{array}{rr}
I & -X \\
0 & I
\end{array}\right)\right],
$$

is bijective and satisfies

$$
\kappa(M\langle Z\rangle)=\kappa(Z)\left[M^{-1}\right]
$$

for all $M \in \operatorname{MSp}(n ; \mathbf{F})$ and $Z \in \mathscr{H}(n ; \mathbf{F})$.

Proof. $\kappa(Z) \in \mathscr{P}(n ; \mathbf{F})$ follows from (1.1). The surjectivity of $\kappa$ is obtained by the method of completing squares (cf. [16], I.3.2). Since $\kappa$ is obviously injective, the first part is proved.

In order to demonstrate $(*)$ we may confine ourselves to $\mathbf{F}=\mathbf{H}$ and to the generators (1.4) of $\operatorname{MSp}(n ; \mathbf{H})$. An explicit calculation using Theorem 1.3 completes the proof.

There also exists a bounded domain, which is birationally equivalent to the half-space. Consider the generalized unit disc

$$
\mathscr{D}(n ; \mathbf{F}):=\left\{W \in \operatorname{Mat}(n ; \mathbf{F}) ; I-\bar{W}^{\prime} W \in \operatorname{Pos}(n ; \mathbf{F})\right\} .
$$

The generalized Cayley transformation yields that the maps

$$
\begin{array}{rlrl}
\mathscr{H}(n ; \mathbf{F}) \rightarrow \mathscr{D}(n ; \mathbf{F}), & & Z \mapsto(Z-I)(Z+I)^{-1}, \\
\mathscr{D}(n ; \mathbf{F}) \rightarrow \mathscr{H}(n ; \mathbf{F}), & W \mapsto(W+I)(-W+I)^{-1},
\end{array}
$$

are bijective and inverse to each other. 
As a consequence one obtains a good description of the stabilizer

$$
\operatorname{Stab}(Z):=\{M \in \operatorname{MSp}(n ; \mathbf{F}) ; M\langle Z\rangle=Z\}, \quad Z \in \mathscr{H}(n ; \mathbf{F}) .
$$

We need the unitary group

$$
\mathscr{U}(n ; \mathbf{F}):=\left\{U \in \operatorname{Mat}(n ; \mathbf{F}) ; \bar{U}^{\prime} U=U \bar{U}^{\prime}=I\right\} .
$$

Then an explicit calculation yields

Proposition 1.6.

$$
\begin{aligned}
& \operatorname{Stab}(I)=\operatorname{MSp}(n ; \mathbf{F}) \cap \mathscr{U}(2 n ; \mathbf{F}) \\
& \quad=\left\{\left(\begin{array}{ll}
A & B \\
B & A
\end{array}\right) ; A, B \in \operatorname{Mat}(n ; \mathbf{F}), A \bar{B}^{\prime}+B \bar{A}^{\prime}=0, A \bar{A}^{\prime}+B \bar{B}^{\prime}=I\right\} \\
& \quad=\left\{\frac{1}{2}\left(\begin{array}{cc}
I & I \\
-I & I
\end{array}\right)\left(\begin{array}{cc}
U & 0 \\
0 & V
\end{array}\right)\left(\begin{array}{cc}
I & -I \\
I & I
\end{array}\right) ; U, V \in \mathscr{U}(n ; \mathbf{F})\right\} .
\end{aligned}
$$

REMARK 1.7. Consider the three-dimensional hyperbolic space

$$
\mathscr{H}=\left\{z=\sum_{j=1}^{3} z_{j} e_{j} ; z_{j} \in \mathbf{R}, z_{3}>0\right\}
$$

investigated in [6]. Clearly $\mathscr{H}$ becomes a real submanifold of

$$
e_{3} \cdot \mathscr{H}(1 ; \mathbf{H})=\left\{z=\sum_{j=1}^{4} z_{j} e_{j} ; z_{j} \in \mathbf{R}, z_{3}>0\right\} .
$$

In view of $(0.3)$ one easily verifies that the group

$$
\Sigma=\left(\begin{array}{cc}
e_{3} & 0 \\
0 & 1
\end{array}\right) \operatorname{MSp}(1 ; \mathbf{H})\left(\begin{array}{cc}
e_{3} & 0 \\
0 & 1
\end{array}\right)^{-1}
$$

contains $\operatorname{SL}(2 ; \mathrm{C})$ as a subgroup. Now one can show that

$$
\{M \in \Sigma ; M\langle\mathscr{H}\rangle=\mathscr{H}\}=\operatorname{SL}(2 ; \mathbf{C}) \cup\left(e_{3} I\right) \cdot \operatorname{SL}(2 ; \mathbf{C}) .
$$

The right-hand side proves to be a group by virtue of $\left(e_{3} I\right) \cdot M$. $\left(e_{3} I\right)^{-1}=\bar{M}$ for $M \in \operatorname{Mat}(2 ; \mathbf{C})$. Moreover, note that $z=z_{1} e_{1}+$ $z_{2} e_{2}+z_{3} e_{3} \in \mathscr{H}$ implies

$$
\left(e_{3} I\right)\langle z\rangle=z_{1} e_{1}-z_{2} e_{2}+z_{3} e_{3} .
$$

2. The half-space as a symmetric space. One can proceed in the same way, as C. L. Siegel [29] did in the classical situation, in order to turn the half-space into a symmetric space. 
Given $Z, W \in \operatorname{Mat}(n ; \mathbf{F}), Z=\left(z_{k l}\right), z_{k l}=\sum_{j=1}^{r} z_{k l}^{(j)} \boldsymbol{e}_{j}, z_{k l}^{(j)} \in \mathbf{R}$, set $\tau(Z, W):=\frac{1}{2} \operatorname{trace}\left(Z \bar{W}^{\prime}+W \bar{Z}^{\prime}\right)$ and let $d Z$ denote the matrix of differentials

$$
d Z=\left(\sum_{j=1}^{r} d z_{k l}^{(j)} e_{j}\right)_{1 \leq k, l \leq n} .
$$

Now consider the quadratic differential form

$$
d s^{2}:=\tau\left(Y^{-1} d Z Y^{-1}, d Z\right)
$$

whenever $Z=X+Y \in \mathscr{H}(n ; \mathbf{F})$. The case $\mathbf{F}=\mathbf{C}$ of the following assertion is due to $\mathrm{H}$. Braun [3].

LemMa 2.1. The quadratic differential form $d s^{2}$ is positive definite in $\mathscr{H}(n ; \mathbf{F})$ and invariant under the maps $Z \mapsto M\langle Z\rangle, M \in \operatorname{MSp}(n ; \mathbf{F})$, as well as $Z \mapsto \bar{Z}^{\prime}$.

Proof. $\tau(A, B)=\tau\left(\bar{A}^{\prime}, \bar{B}^{\prime}\right)$ yields the invariance under $Z \mapsto \bar{Z}^{\prime}$. Let $M \in \operatorname{MSp}(n ; \mathbf{F}), Z \in \mathscr{H}(n ; \mathbf{F})$ and set $Z_{1}=M\langle Z\rangle$. Then (1.1) and Proposition 1.4 lead to

$$
d Z_{1}={\overline{\tilde{M}}\left\{\bar{Z}^{\prime}\right\}}^{\prime-1} d Z(M\{Z\})^{-1} .
$$

Next $\left.Y_{1}=(M\{Z\}) Y^{-1} \bar{M}\{Z\}^{\prime}=\left(\tilde{M}\left\{\bar{Z}^{\prime}\right\}\right) Y^{-1} \overline{\tilde{M}\left\{\bar{Z}^{\prime}\right.}\right\}$ follows from Theorem 1.3 and Proposition 1.4. Finally, the use of [16], IV.1.1, yields

$$
\tau\left(Y_{1}^{-1} d Z_{1} Y_{1}^{-1}, d Z_{1}\right)=\tau\left(Y^{-1} d Z Y^{-1}, d Z\right) .
$$

$d s^{2}$ is obviously positive definite in the point $Z=I$. Since $\operatorname{MSp}(n ; \mathbf{F})$ acts transitively, the assertion follows.

In Helgason's notation [8] we obtain

THeOREM 2.2. $\mathscr{H}(n ; \mathbf{F})$ endowed with the metric $d s^{2}$ is a Riemannian globally symmetric space of the noncompact type, which is irreducible except for the cases $\mathbf{F}=\mathbf{R}, n=1,2$.

Proof. The map $Z \mapsto Q\langle Z\rangle=Z^{-1}$ becomes an involutive isometry, which possesses $I$ as an isolated fixed point.

With the aid of Proposition 1.6 we determine the associated Lie algebras, namely

$$
\begin{aligned}
\text { Lie } \operatorname{MSp}(n ; \mathbf{F}) & =\left\{M \in \operatorname{Mat}(2 n ; \mathbf{F}) ; \bar{M}^{\prime} Q+Q M=0\right\} \\
& =\left\{\left(\begin{array}{cc}
A & B \\
C & -\bar{A}^{\prime}
\end{array}\right) ; A \in \operatorname{Mat}(n ; \mathbf{F}), B, C \in \operatorname{Alt}(n ; \mathbf{F})\right\},
\end{aligned}
$$

Lie $\operatorname{Stab}(I)=\operatorname{Lie} \operatorname{MSp}(n ; \mathbf{F}) \cap \operatorname{Alt}(2 n ; \mathbf{F})$. 
Now one easily checks

$$
\begin{aligned}
& \left(\begin{array}{rr}
I & I \\
-I & I
\end{array}\right) \text { Lie } \operatorname{MSp}(n ; \mathbf{F})\left(\begin{array}{rr}
I & I \\
-I & I
\end{array}\right)^{-1}=\left\{\begin{array}{cc}
\mathfrak{s} \mathfrak{o}(n, n) & \text { if } \mathbf{F}=\mathbf{R}, \\
\mathfrak{u}(n, n) & \text { if } \mathbf{F}=\mathbf{C},
\end{array}\right. \\
& \left(\begin{array}{rr}
I & I \\
-I & I
\end{array}\right) \text { Lie } \operatorname{Stab}(I)\left(\begin{array}{rr}
I & I \\
-I & I
\end{array}\right)^{-1}=\left\{\begin{array}{cc}
\mathfrak{s o}(n) \times \mathfrak{s o}(n) & \text { if } \mathbf{F}=\mathbf{R}, \\
\mathfrak{u}(n) \times \mathfrak{u}(n) & \text { if } \mathbf{F}=\mathbf{C},
\end{array}\right.
\end{aligned}
$$

(cf. [8], p. 341). In the case $\mathbf{F}=\mathbf{H}$ a similar map yields an isomorphism between Lie $\operatorname{MSp}(n ; \mathbf{H})$ and $\mathfrak{s p}(n, n)$ as well as between Lie $\operatorname{Stab}(I)$ and $\mathfrak{s p}(n) \times \mathfrak{s p}(n)$. Now the assertion follows from Helgason's classification (cf. [8], IX,§4).

REMARK 2.3. (a) $\mathscr{H}(n ; \mathbf{F})$ corresponds to BDI for $\mathbf{F}=\mathbf{R}$, to AIII for $\mathbf{F}=\mathbf{C}$ and to $\mathbf{C I I}$ for $\mathbf{F}=\mathbf{H}$ in Helgason's classification (cf. [8], p. 354), where in every case $p=q=n$. Note that the spaces $\mathscr{H}(n ; \mathbf{R}), n \neq 2$, and $\mathscr{H}(n ; \mathbf{H}), n \geq 1$, fail to be Hermitian (cf. [8], p. 354).

(b) In view of $[8]$, p. $353,(\mathbf{x})$, the space $\mathscr{H}(2 ; \mathbf{R})$ is isomorphic to the direct product of two copies of the upper half-plane $\mathscr{H}=\{z=$ $x+i y \in \mathbf{C} ; y>0\}$ in $\mathbf{C}$. Each $Z \in \mathscr{H}(2 ; \mathbf{R})$ is uniquely representable as

Now define the map

$$
Z=x J+Y=\left(\begin{array}{ll}
y_{1} & y+x \\
y-x & y_{2}
\end{array}\right) \text {. }
$$

$$
\chi_{2}: \mathscr{H}(2 ; \mathbf{R}) \rightarrow \mathscr{H} \times \mathscr{H}, \quad Z \mapsto\left(x+i \sqrt{\operatorname{det} Y}, \frac{1}{y_{1}}(-y+i \sqrt{\operatorname{det} Y})\right) .
$$

Clearly $\chi_{2}$ becomes a bijection. If $\chi_{2}(Z)=(z, w)$ and $U \in \operatorname{GL}(2 ; \mathbf{R})$ one easily verifies

$$
\begin{aligned}
& \chi_{2}(Z+J)=(z+1, w), \\
& \chi_{2}\left(U^{\prime} Z U\right)= \begin{cases}\left(\operatorname{det} U \cdot z, U^{-1}\langle w\rangle\right) & \text { if } \operatorname{det} U>0, \\
\left(\operatorname{det} U \cdot \bar{z}, U^{-1}\langle\bar{w}\rangle\right) & \text { if } \operatorname{det} U<0,\end{cases} \\
& \chi_{2}\left(Z^{-1}\right)=\left(-\frac{1}{z},-\frac{1}{w}\right), \\
& \chi_{2}((Q \times I)\langle Z\rangle)=(w, z), \quad \text { where } Q=Q^{(2)}, \quad I=I^{(2)} .
\end{aligned}
$$

(c) In view of $[8]$, p. 352 , (iv), the space $\mathscr{H}(3 ; \mathbf{R})$ is isomorphic to the space $\operatorname{SPos}(4 ; \mathbf{R})=\operatorname{Pos}(4 ; \mathbf{R}) \cap \operatorname{SL}(4 ; \mathbf{R})$ (cf. [32]). Given $x=$ $\left(x_{1}, x_{2}, x_{3}\right)^{\prime} \in \mathbf{R}^{3}$ we define

$$
\operatorname{ad} x=\left(\begin{array}{ccc}
0 & -x_{3} & x_{2} \\
x_{3} & 0 & -x_{1} \\
-x_{2} & x_{1} & 0
\end{array}\right) \in \operatorname{Alt}(3 ; \mathbf{R})
$$


which comes from the vector product (cf. [15], p. 205). Now set

$$
\begin{gathered}
\chi_{3}: \mathscr{H}(3 ; \mathbf{R}) \rightarrow \operatorname{SPos}(4 ; \mathbf{R}) \\
\operatorname{ad} x+Y \mapsto(\operatorname{det} Y)^{-1 / 2}\left(\begin{array}{cc}
Y & 0 \\
0 & \operatorname{det} Y
\end{array}\right)\left[\left(\begin{array}{cc}
I & x \\
0 & 1
\end{array}\right)\right] .
\end{gathered}
$$

Given $s \in \mathbf{R}^{3}, U \in \mathrm{GL}(3 ; \mathbf{R})$ one easily verifies

$$
\begin{aligned}
& \chi_{3}(Z+\operatorname{ad} s)=\chi_{3}(Z)\left[\left(\begin{array}{ll}
I & s \\
0 & 1
\end{array}\right)\right], \\
& \chi_{3}\left(U^{\prime} Z U\right)=\chi_{3}(Z)\left[U^{*}\right], \quad \text { where } U^{*}=|\operatorname{det} U|^{-1 / 2}\left(\begin{array}{cc}
U & 0 \\
0 & \operatorname{det} U
\end{array}\right), \\
& \chi_{3}\left(Z^{-1}\right)=\left(\chi_{3}(Z)\right)^{-1} .
\end{aligned}
$$

Now we are going to describe the associated invariant volume element and the Laplace-Beltrami-operator, which was determined by H. Maaß [21] in the case of the Siegel half-space. Therefore define the vector

$$
d \mathfrak{z}=\left(d z_{11}^{(1)}, \ldots, d z_{11}^{(r)}, d z_{12}^{(1)}, \ldots, d z_{1 n}^{(r)}, d z_{21}^{(1)}, \ldots, d z_{n n}^{(r)}\right)^{\prime}
$$

of the length $r n^{2}$. Given $Y \in \operatorname{Pos}(n ; \mathbf{F})$ there exists $S_{Y} \in \operatorname{Pos}\left(r n^{2} ; \mathbf{R}\right)$ satisfying

$$
d s^{2}=\tau\left(Y^{-1} d Z Y^{-1}, d Z\right)=S_{Y}[d \mathfrak{z}]
$$

in view of Lemma 2.1.

Proposition 2.4. The volume element

$$
d v=(\operatorname{det} Y)^{-r n} \prod_{k=1}^{n} \prod_{l=1}^{n} \prod_{j=1}^{r} d z_{k l}^{(j)}
$$

of $\mathscr{H}(n ; \mathbf{F})$ is invariant under the modified symplectic transformations $Z \mapsto M\langle Z\rangle, M \in \operatorname{MSp}(n ; \mathbf{F})$, as well as $Z \mapsto \bar{Z}^{\prime}$.

Proof. Define $d:=\operatorname{det} S_{Y}$; then $d v=d^{1 / 2} \prod_{k, l, j} d z_{k l}^{(j)}$ has the desired invariance property due to Lemma 2.1. One calculates $d=$ $(\operatorname{det} Y)^{-2 r n}$.

We compute the effect of differential operators on determinants. 
Proposition 2.5. Let $Y \in \operatorname{Pos}(n ; \mathbf{F}), Y^{-1}=\left(\tilde{y}_{k l}\right)$ and $s \in \mathbf{C}$. Given $1 \leq k, l \leq n, 1 \leq j \leq r$, one has

$$
\frac{\partial}{\partial z_{k l}^{(j)}}(\operatorname{det} Y)^{s}=s(\operatorname{det} Y)^{s} \tilde{y}_{k l}^{(j)}
$$

Proof. Due to the method of completing squares (cf. [16], I.3.2), we may confine ourselves to the case $n=2$. Then an explicit calculation completes the proof.

In order to get an explicit description of the Laplace-Beltrami-operator, let $\partial / \partial Z$ denote the matrix differential operator

$$
\frac{\partial}{\partial z}=\left(\sum_{j=1}^{r} \frac{\partial}{\partial z_{k l}^{(j)}} e_{j}\right)_{1 \leq k, l \leq n}
$$

THEOREM 2.6. The Laplace-Beltrami-operator $\Delta$ is invariant under the maps $Z \mapsto M\langle Z\rangle, M \in \operatorname{MSp}(n ; \mathbf{F})$, as well as $Z \mapsto \bar{Z}^{\prime}$ and is given by

$$
\Delta=\tau\left(Y \frac{\partial}{\partial Z} Y, \frac{\partial}{\partial Z}\right)-\left(\frac{1}{2} r(n+1)-1\right) \tau\left(Y, \frac{\partial}{\partial Z}\right) .
$$

Proof. The invariance follows from Lemma 2.1 and [8], X.2.1. Using (2.1) an elementary but lengthy calculation yields $\left(S_{Y}\right)^{-1}=S_{Y^{-1}}$. Then the definition of $\Delta$ leads to

$$
\Delta=\sum_{\substack{1 \leq j, k, l, m \leq n \\ 1 \leq \nu, \mu \leq r}}(\operatorname{det} Y)^{r n} \frac{\partial}{\partial z_{k l}^{(\nu)}} \operatorname{Re}\left(y_{j k} e_{\nu} y_{l m} \bar{e}_{\mu}\right)(\operatorname{det} Y)^{-r n} \frac{\partial}{\partial z_{j m}^{(\mu)}}
$$

Now one can use Proposition 2.5 and another lengthy calculation shows that $\Delta$ has the form given above.

Theorem 2.6 combined with Proposition 2.5 yields

Corollary 2.7. Let $Z \in \mathscr{H}(n ; \mathbf{F}), M \in \operatorname{MSp}(n ; \mathbf{F})$ and $s \in \mathbf{C}$. Then one has

$$
\Delta\left(\operatorname{det} Y_{M}\right)^{s}=n s\left(s+1-\frac{1}{2} r(n+1)\right)\left(\operatorname{det} Y_{M}\right)^{s} .
$$

REMARK 2.8. One can proceed in the same way as C. L. Siegel [29], resp. $H$. Klingen [12], in order to derive normal forms for pairs of points under modified symplectic transformations. As a result one 
obtains that the geodesics in $\mathscr{H}(n ; \mathbf{F})$ are given by the images of the curves

$$
Z(u)=\left(\begin{array}{lll}
e^{u p_{1}} & & 0 \\
& \ddots & \\
0 & & e^{u p_{n}}
\end{array}\right)
$$

under the transformations $Z \mapsto M\langle Z\rangle, M \in \operatorname{MSp}(n ; \mathbf{F})$. Here $p_{1}, \ldots, p_{n}$ satisfy $0 \leq p_{1} \leq \cdots \leq p_{n}$ as well as $\sum_{k=1}^{n} p_{k}^{2}=1$ and $u$ runs through the interval $[0, \rho]$, where $\rho$ denotes the geodesic distance of the points. On the other hand the geodesics in $\mathscr{H}(n ; \mathbf{F})$ coincide with the solutions of the differential equation

$$
\ddot{Z}=\dot{Z} Y^{-1} \dot{Z} \text {. }
$$

Thus in the relations

$$
\mathscr{H}(n ; \mathbf{R}) \subset \mathscr{H}(n ; \mathbf{C}) \subset \mathscr{H}(n ; \mathbf{H})
$$

every half-space becomes a totally geodesic submanifold of the following one.

3. The modified modular group. We proceed in the same way as in [16]. Thus we obtain integral elements by the choice of a special order $\mathscr{O}=\mathscr{O}(\mathbf{F})$, namely

$$
\mathscr{O}(\mathbf{R})=\mathbf{Z}, \quad \mathscr{O}(\mathbf{C})=\mathbf{Z} e_{1}=\mathbf{Z} e_{2}, \quad \mathscr{O}(\mathbf{H})=\mathbf{Z} e_{0}+\mathbf{Z} e_{1}+\mathbf{Z} e_{2}+\mathbf{Z} e_{3},
$$

where $e_{0}=\frac{1}{2}\left(e_{1}+e_{2}+e_{3}+e_{4}\right)$. Here $\mathscr{O}(\mathbf{C})$ of course denotes the Gaussian integers and $\mathscr{O}(\mathbf{H})$ the quaternions of Hurwitz (cf. [9] or [5], $\S 91)$. Then the set of integral modified symplectic matrices

$$
\Gamma(n ; \mathscr{O}):=\operatorname{MSp}(n ; \mathbf{F}) \cap \operatorname{Mat}(2 n ; \mathscr{O})
$$

becomes a subgroup of $\operatorname{MSp}(n ; \mathbf{F})$, which acts discontinuously on the half-space $\mathscr{H}(n ; \mathbf{F})$.

DEFINITION. $\Gamma(n ; \mathscr{O})$ is called the modified modular group of degree n.

Clearly, we include the trivial case

$$
\Gamma(1 ; \mathbf{Z})=\{ \pm I, \pm Q\}
$$

in view of (1.3). In the case $\mathbf{F}=\mathbf{C}(0.2)$ implies that

$$
\left(\begin{array}{ll}
e_{2} I & 0 \\
0 & I
\end{array}\right) \Gamma\left(n ; \mathbf{Z} e_{1}+\mathbf{Z} e_{2}\right)\left(\begin{array}{ll}
e_{2} I & 0 \\
0 & I
\end{array}\right)^{-1}
$$


equals the Hermitian modular group with respect to the Gaussian number field (cf. [3]).

Let $\operatorname{Alt}(n ; \mathscr{O})$ denote the lattice of all integral skew-Hermitian $n \times n$ matrices. $\operatorname{GL}(n ; \mathscr{O})$ stands for the group of units in the $\operatorname{ring} \operatorname{Mat}(n ; \mathscr{O})$. Thus (1.5) yields

$$
\begin{aligned}
\Gamma(n ; \mathscr{O})_{\infty}: & =\operatorname{MSp}(n ; \mathbf{F})_{\infty} \cap \operatorname{Mat}(2 n ; \mathscr{O}) \\
& =\left\{\left(\begin{array}{cc}
\bar{U}^{\prime} & \bar{U}^{\prime} S \\
0 & U^{-1}
\end{array}\right) ; U \in \operatorname{GL}(n ; \mathscr{O}), S \in \operatorname{Alt}(n ; \mathscr{O})\right\}
\end{aligned}
$$

Set $N(a):=a \bar{a} \in \mathbf{R}$ for $a \in \mathbf{F}$. Hence one easily verifies the property:

Given $a \in \operatorname{Alt}(1 ; \mathbf{F})$ then $g \in \operatorname{Alt}(1 ; \mathscr{O})$ exists such that $N(a-g)<1$.

Hence the Euclidean algorithm is valid in $\mathscr{O}$ as well as in $\operatorname{Alt}(1 ; \mathscr{O})$. Thus we can derive a result of L. Kronecker [18] —often cited as Witt's Theorem [33] - on the generators of the modified modular group. The proofs in [16], II.2.2 and II.2.3, can be adapted by the use of (1.1) and (3.4) in order to obtain

THEOREM 3.1. The modified modular group $\Gamma(n ; \mathscr{O})$ is generated by the matrices

$Q^{(2)} \times I, \quad\left(\begin{array}{ll}I & S \\ 0 & I\end{array}\right), \quad S \in \operatorname{Alt}(n ; \mathscr{O}), \quad\left(\begin{array}{ll}\bar{U}^{\prime} & 0 \\ 0 & U^{-1}\end{array}\right), \quad U \in \operatorname{GL}(n ; \mathscr{O})$.

The same arguments that were applied in the proof of Lemma 1.1b yield that $\Gamma(n ; \mathscr{O})$ can also be generated by the matrices

$$
Q, \quad\left(\begin{array}{ll}
I & S \\
0 & I
\end{array}\right), \quad S \in \operatorname{Alt}(n ; \mathscr{O}), \quad\left(\begin{array}{ll}
\bar{U}^{\prime} & 0 \\
0 & U^{-1}
\end{array}\right), \quad U \in \mathrm{GL}(n ; \mathscr{O}),
$$

except for the case $\mathscr{O}=\mathbf{Z}, n$ even.

Combining this with (1.8) it becomes clear that the group $\Delta_{n}^{*}$ considered by $\mathrm{H}$. Maaß in [23] equals $\Gamma(n ; \mathbf{Z})$, whenever $n$ is odd, and $\Gamma(n ; \mathbf{Z}) \cap \operatorname{SL}(2 n ; \mathbf{Z})$, whenever $n$ is even.

Now we are going to determine a suitable fundamental domain. Therefore let $\mathscr{C}(n ; \mathscr{O})$ denote the fundamental parallelotope of the lattice $\operatorname{Alt}(n ; \mathscr{O})$ in $\operatorname{Alt}(n ; \mathbf{F})$, which consists of the matrices $X=\left(x_{k l}\right) \in$ $\operatorname{Alt}(n ; \mathbf{F})$ such that

$$
x_{k l}=\sum_{j=1}^{r} x_{k l}^{(j)} e_{j}, \quad-\frac{1}{2} \leq x_{k l}^{(j)} \leq \frac{1}{2}, 1 \leq k \leq l \leq n, 1 \leq j \leq r,
$$


where $x_{k l}^{(1)} \geq 0$ in the case $\mathbf{F}=\mathbf{H}$. Moreover, $\mathscr{R}(n ; \mathbf{F})$ stands for the set of reduced matrices in $\operatorname{Pos}(n ; \mathbf{F})$ (cf. [16], p. 29). Now let $\mathscr{F}(n ; \mathscr{O})$ consist of all matrices $Z=X+Y \in \mathscr{H}(n ; \mathbf{F})$, which satisfy

(i) $X \in \mathscr{C}(n ; \mathscr{O})$,

(ii) $Y \in \mathscr{R}(n ; \mathbf{F})$,

(iii) $|\operatorname{det} M\{Z\}| \geq 1$, i.e. $\operatorname{det} Y_{M} \leq \operatorname{det} Y$, for all $M \in \Gamma(n ; \mathscr{O})$. Clearly, one has

$$
\begin{gathered}
\mathscr{F}(1 ; \mathbf{Z})=\{y \in \mathbf{R} ; y \geq 1\}, \\
i \mathscr{F}\left(n ; \mathbf{Z} e_{1}+\mathbf{Z} e_{2}\right)=\mathscr{F}(n ; \mathbf{C}),
\end{gathered}
$$

where $\mathscr{F}(n ; \mathbf{C})$ denotes the fundamental domain in [3] resp. [16], p. 58. At first we derive some properties of the domain $\mathscr{F}(n ; \mathscr{O})$.

Proposition 3.2. There exists a constant $\rho=\rho(n ; \mathbf{F})$ such that $Y \geq \rho I$ holds for all $Z=X+Y \in \mathscr{F}(n ; \mathscr{O})$.

Proof. $1 \leq\left|\operatorname{det}\left(Q^{(2)} \times I\right)\{Z\}\right|^{2}=N\left(z_{11}\right)=y_{11}^{2}+N\left(x_{11}\right)$ holds in view of (iii). The definition of $\mathscr{C}(n ; \mathscr{O})$ yields $N\left(x_{11}\right) \leq \frac{3}{4}$, hence $y_{11} \geq \frac{1}{2}$. Now [16], I.4.7 and I.5.1, combined with (ii) imply $Y \geq \frac{1}{2} \beta I$, where $\beta$ only depends on $n$.

Let $d v$ again denote the invariant volume element (cf. Proposition 2.4). One can apply nearly the same arguments, which were used for the proof of [16], II.3.2, II.3.9, in order to obtain

LEMMA 3.3. (a) $\lambda I \in \mathscr{F}(n ; \mathscr{O})$ for all $\lambda \geq 1$.

(b) Given $Z=X+Y \in \mathscr{F}(n ; \mathscr{O})$, then $Z_{\lambda}:=X+\lambda Y \in \mathscr{F}(n ; \mathscr{O})$ holds for $\lambda \geq 1$.

(c) $\mathscr{F}(n ; \mathscr{O})$ is arcwise connected.

(d) $\operatorname{vol}(\mathscr{F}(n ; \mathscr{O})):=\int_{\mathscr{F}(n ; \mathcal{O})} d v<\infty$ except for $n=1, \mathscr{O}=\mathbf{Z}$.

Hence the domain $\mathscr{F}(n ; \mathscr{O})$ fails to be compact. Given $\alpha>0$ the subset $\mathscr{E}(n ; \mathbf{F})[\alpha]$ of $\operatorname{Pos}(n ; \mathbf{F})$ consists of the matrices

$$
\left(\begin{array}{ccc}
d_{1} & & 0 \\
& \ddots & \\
0 & & d_{n}
\end{array}\right)\left[\left(\begin{array}{ccc}
1 & & b_{k l} \\
& \ddots & \\
0 & & 1
\end{array}\right)\right],
$$

where $0<d_{j}<\alpha d_{j+1}$ for $1 \leq j<n$ and $N\left(b_{k l}\right)<\alpha^{2}$ for $1 \leq k<l \leq n$ (cf. [16], p. 33). Then we define the Siegel set

$\mathscr{S}(n ; \mathbf{F})[\alpha]:=\left\{Z \in \mathscr{H}(n ; \mathbf{F}) ; N\left(x_{k l}\right)<\alpha^{2}, Y \in \mathscr{E}(n ; \mathbf{F})[\alpha], 1<\alpha y_{11}\right\}$, 
confer [7], p. 90, in the case of the Siegel half-space. Recall the definition of $\kappa$ from Theorem 1.5 and consider the matrices

$V_{0}=\left(\begin{array}{ccc}0 & & 1 \\ & . \cdot & \\ 1 & & 0\end{array}\right) \in \mathrm{GL}(n ; \mathscr{O}) \quad$ and $\quad W_{0}=\left(\begin{array}{ll}V_{0} & 0 \\ 0 & I\end{array}\right) \in \mathrm{GL}(2 n ; \mathscr{O})$.

LEMMA 3.4. (a) There exists $\alpha=\alpha(n ; \mathbf{F})>0$ such that

$$
\mathscr{F}(n ; \mathscr{O}) \subset \mathscr{S}(n ; \mathbf{F})[\alpha] .
$$

(b) Given a compact subset $\mathscr{C}$ in $\mathscr{H}(n ; \mathbf{F})$, there exists $\beta=\beta(\mathscr{C})>0$ satisfying

$$
\mathscr{C} \subset \mathscr{S}(n ; \mathbf{F})[\beta] \text {. }
$$

(c) Given $\gamma>0$ one can find $\delta>0$ such that

$$
\kappa(\mathscr{S}(n ; \mathbf{F})[\gamma])\left[W_{0}\right] \subset \mathscr{E}(2 n ; \mathbf{F})[\delta] .
$$

(d) Let $\gamma>0$, then there are only finitely many $M \in \Gamma(n ; \mathscr{O})$ satisfying

$$
M\langle\mathscr{S}(n ; \mathbf{F})[\gamma]\rangle \cap \mathscr{S}(n ; \mathbf{F})[\gamma] \neq \varnothing .
$$

Proof. (a) and (b) The proof is settled in analogy with [16], II. 3.6, where Proposition 3.2 is applied.

(c) Proceed in the same way as in [16], II.3.7.

(d) The assertion follows from part (c) combined with [16], I.4.10.

We take the definition of a fundamental domain from [16], p. 6.

THeOREM 3.5. $\mathscr{F}(n ; \mathscr{O})$ is a fundamental domain of $\mathscr{H}(n ; \mathbf{F})$ with respect to the action of $\Gamma(n ; \mathscr{O})$ except for $\mathbf{F}=\mathbf{H}, n=1$. The domain $\mathscr{F}(n ; \mathscr{O})$ is arcwise connected and closed in $\operatorname{Mat}(n ; \mathbf{F})$. Moreover $\operatorname{vol}(\mathscr{F}(n ; \mathscr{O}))<\infty$ holds except for $\mathbf{F}=\mathbf{R}, n=1$.

Proof. Given $Z \in \mathscr{H}(n ; \mathbf{F})$ we can show in the same way as in [16], II.3.3, that there exists $M \in \Gamma(n ; \mathscr{O})$ satisfying

$$
\operatorname{det} Y_{K} \leq \operatorname{det} Y_{M} \quad \text { for all } K \in \Gamma(n ; \mathscr{O}) \text {. }
$$

We may replace $M$ by $K M$, where $K \in \Gamma(n ; \mathscr{O})_{\infty}$, in order to map $Z$ into $\mathscr{F}(n ; \mathscr{O})$ by a modified modular transformation.

In view of the definition $\mathscr{F}(n ; \mathscr{O})$ is relatively closed in $\mathscr{H}(n ; \mathbf{F})$. Now $\mathscr{F}(n ; \mathscr{O})$ proves to be closed in $\operatorname{Mat}(n ; \mathbf{F})$ according to Proposition 3.2. By virtue of

$$
\bigcup_{M} M\langle\mathscr{F}(n ; \mathscr{O})\rangle=\mathscr{H}(n ; \mathbf{F})
$$


where $M$ runs through $\Gamma(n ; \mathscr{O})$, clearly $\mathscr{F}(n ; \mathscr{O})$ contains interior points.

Let $M \in \Gamma(n ; \mathscr{O})$ and $Z \in \mathscr{F}(n ; \mathscr{O})$ such that $Z$ and $W:=M\langle Z\rangle$ are interior points of $\mathscr{F}(n ; \mathscr{O})$. We obtain $(M\{Z\})^{-1}=M^{-1}\{W\}$ from Theorem 1.3. Thus $|\operatorname{det} M\{Z\}|=\left|\operatorname{det} M^{-1}\{W\}\right|=1$ follows. Since $Z$ and $W$ are interior points, we conclude $C=0$. Then (3.3) implies

$$
W=Z[U]+S
$$

for appropriate $U \in \mathrm{GL}(n ; \mathscr{O})$ and $S \in \operatorname{Alt}(n ; \mathscr{O})$. Since $Y$ is an interior point of $\mathscr{R}(n ; \mathbf{F})$, whenever $Z=X+Y$, we conclude $U=\varepsilon I$, where $\varepsilon$ is a unit in $\mathscr{O}$ and belongs to the center of $\mathbf{F}$, if $n>1$. Finally we obtain $S=0$, because $X$ lies in the open kernel of $\mathscr{C}(n ; \mathscr{O})$.

The remaining assertions follow from Lemma 3.3 and 3.4.

In the case $\mathbf{F}=\mathbf{H}, n=1$ we observe that the matrices $M=\varepsilon I^{(2)}$, where $\varepsilon \in \mathscr{E}=\{g \in \mathscr{O} ; N(g)=1\}$, induce the identity map on $\operatorname{Pos}(1 ; \mathbf{H})=\mathbf{R}^{+}$. Using [16], I.1.3, and the considerations above, we obtain a fundamental domain $\mathscr{F}^{*}$ of $\mathscr{H}(1 ; \mathbf{H})$ with respect to the action of $\Gamma(1 ; \mathscr{O})$, where

$$
\mathscr{F}^{*}=\left\{z=x+y \in \mathscr{F}(1 ; \mathscr{O}) ; x=\sum_{j=2}^{4} x_{j} e_{j}, x_{2} \geq x_{3} \geq 0, x_{2} \geq\left|x_{4}\right|\right\} \text {. }
$$

But we can simplify the condition (iii) and gain

COROLlaRY 3.6. A fundamental domain of $\mathscr{H}(1 ; \mathbf{H})$ with respect to the action of $\Gamma(1 ; \mathscr{O})$ is given by

$\mathscr{F}^{*}=\left\{z=\sum_{j=1}^{4} z_{j} e_{j} \in \mathbf{H} ; z_{1}>0, \frac{1}{2} \geq z_{2} \geq z_{3} \geq 0, z_{2} \geq\left|z_{4}\right|, N(z) \geq 1\right\}$.

Moreover, besides the obvious cases $n=1, \mathbf{F}=\mathbf{R}, \mathbf{C}$ (cf. (3.5), (3.6)) the domain $\mathscr{F}(2 ; \mathbf{Z})$ can be described easily.

EXAMPLE 3.7. The fundamental domain $\mathscr{F}(2 ; Z)$ consists of the matrices

$$
Z=\left(\begin{array}{ll}
y_{1} & y+x \\
y-x & y_{2}
\end{array}\right) \in \operatorname{Mat}(2 ; \mathbf{R})
$$

where

$$
\begin{gathered}
1 \leq y_{1} \leq y_{2}, \quad 0 \leq 2 y \leq y_{1}, \quad-\frac{1}{2} \leq x \leq \frac{1}{2} \\
\operatorname{det} Z=y_{1} y_{2}-y^{2}+x^{2} \geq 1
\end{gathered}
$$


REMARK 3.8. Let us replace $\Gamma(n ; \mathbf{Z})$ by $\Gamma^{*}(n ; \mathbf{Z}):=\Gamma(n ; \mathbf{Z}) \cap$ $\operatorname{SL}(2 n ; \mathbf{Z})$. In the corresponding fundamental domain $\mathscr{F}^{*}(n ; \mathbf{Z})$ the condition (iii) is only valid for $M \in \Gamma^{*}(n ; \mathbf{Z})$. However $\mathscr{F}^{*}(n ; \mathbf{Z})$ possesses more than one cusp. As an example observe that

$$
\left.\begin{array}{c}
\mathscr{F}^{*}(1 ; \mathbf{Z})=\mathscr{H}(1 ; \mathbf{R})=\mathbf{R}^{+}, \\
\mathscr{F}^{*}(2 ; \mathbf{Z})=\left\{Z=\left(\begin{array}{ll}
y_{1} & y+x \\
y-x & y_{2}
\end{array}\right) \in \mathscr{H}(2 ; \mathbf{R}) ;\right. \\
0 \leq 2 y \leq y_{1} \leq y_{2},-\frac{1}{2} \leq x \leq \frac{1}{2}, \\
\operatorname{det} Z \geq 1
\end{array}\right\} .
$$

In general the diagonal matrix $\left[\frac{1}{\lambda}, \lambda, \ldots, \lambda\right]$ belongs to $\mathscr{F}^{*}(n ; \mathbf{Z})$, whenever $\lambda \geq 1$.

In this special case we can compute the volume of the fundamental domain explicitly.

Proposition 3.9. $\operatorname{vol}(\mathscr{F}(2 ; \mathbf{Z}))=\pi^{2} / 9$.

Proof. In view of Example 3.7 and Remark 3.8 one has

$$
\operatorname{vol}(\mathscr{F}(2 ; \mathbf{Z}))=\frac{1}{4} \int_{\mathscr{D}} d \nu
$$

where

$$
\begin{aligned}
& \mathscr{D}=\left\{Z=\left(\begin{array}{ll}
y_{1} & y+x \\
y-x & y_{2}
\end{array}\right) \in \mathscr{H}(2 ; \mathbf{R})\right. \\
& \left.\qquad 0 \leq|2 y| \leq y_{1} \leq y_{2},|x| \leq \frac{1}{2}, \operatorname{det} Z \geq 1\right\} .
\end{aligned}
$$

Remark 2.3 yields

$$
\chi_{2}(\mathscr{D})=\mathscr{F} \times \mathscr{F}, \quad \mathscr{F}=\left\{x+i y \in \mathbf{C} ; y>0,|x| \leq \frac{1}{2},|z| \geq 1\right\} .
$$

Change of variables leads to

$$
\operatorname{vol}(\mathscr{F}(2 ; \mathbf{Z}))=\left(\int_{\mathscr{F}} y^{-2} d x d y\right)^{2}=\frac{\pi^{2}}{9}
$$

4. Eisenstein-series. We are going to define non-analytic Eisensteinseries in analogy with the classical case, cf. [19], [20]. Special attention is devoted to the behavior of convergence, which is investigated after the model of Eisenstein-series on the Siegel half-space. 
Definition. Given $\varepsilon>0$ the set

$$
\mathscr{V S}_{\varepsilon}(n ; \mathbf{F}):=\left\{Z=X+Y \in \mathscr{H}(n ; \mathbf{F}) ; Y \geq \varepsilon I, \varepsilon^{-2} I \geq \bar{X}^{\prime} X\right\}
$$

is called a vertical strip of height $\varepsilon$.

Using (1.9), (1.10), (1.12) as well as the definition of a vertical strip $\mathscr{V}_{\varepsilon}(n ; \mathbf{F})$ in $H(n ; \mathbf{F})$ (cf. [16], p. 148), we obtain

$$
\begin{gathered}
\mathscr{V S}_{\varepsilon}(n ; \mathbf{R}) \subset \mathscr{V}_{\varepsilon}(n ; \mathbf{C}) \subset \mathscr{V}_{\varepsilon}(n ; \mathbf{H}), \\
i \mathscr{V} \mathscr{S}_{\varepsilon}(n ; \mathbf{C})=\mathscr{V}_{\varepsilon}(n ; \mathbf{C}), \\
\left\{i \check{Z} ; Z \in \mathscr{V} S_{\varepsilon}(n ; \mathbf{H})\right\} \subset \mathscr{V}_{\varepsilon}(2 n ; \mathbf{C}) .
\end{gathered}
$$

Proposition 4.1. Given $\varepsilon>0$ there exists $c=c(n ; \varepsilon)>0$ such that $|\operatorname{det} M\{Z\}| \geq c|\operatorname{det} M\{I\}|$ holds for all $Z \in \mathscr{V S S}_{\varepsilon}(n ; \mathbf{F})$ and $M \in \operatorname{MSp}(n ; \mathbf{F})$.

Proof. In view of (4.1) and (1.12) we may restrict to the case $\mathbf{F}=\mathbf{H}$. Now apply (4.3), (1.11) and [16], V.2.5.

Analogous arguments using [16], V.2.7, and Theorem 1.3 yield

Proposition 4.2. Given a compact subset $\mathscr{C}$ in $\mathscr{H}(n ; \mathbf{F})$ there exists a constant $c=c(\mathscr{C})$ such that all $Z=X+Y, W=U+V \in \mathscr{C}$ and $M \in \operatorname{MSp}(n ; \mathbf{F})$ satisfy

$$
\operatorname{det} Y_{M} \leq c \cdot \operatorname{det} V_{M}
$$

We use the abbreviations

$$
\Gamma_{n}:=\Gamma(n ; \mathscr{O}) \quad \text { and } \quad \Gamma_{n}^{\infty}:=\Gamma(n ; \mathscr{O})_{\infty} .
$$

LemmA 4.3. Let $\varepsilon \in \mathbf{R}, \varepsilon>0$ and $k \in \mathbf{R}, k>r(n+1)-2$. Then the series

$$
\sum_{M: \Gamma_{n}^{\infty} \backslash \Gamma_{n}}|\operatorname{det} M\{Z\}|^{-k}
$$

converges uniformly for $Z \in \mathscr{V}_{\varepsilon}(n ; \mathbf{F})$.

Proof. In view of (3.3) the definition does not depend on the choice of the representatives. Hence let $\mathscr{R}$ denote a fixed set of representatives. According to Proposition 4.1 the series is uniformly majorized by

$$
\sum_{M \in \mathscr{R}}|\operatorname{det} M\{I\}|^{-k} .
$$


Observe that $|\operatorname{det} M\{I\}|^{-2}=\operatorname{det} Y$, whenever $M\langle I\rangle=X+Y$. Let $d v$ denote the invariant volume element quoted in Proposition 2.4. Moreover set

$$
\mathscr{C}=\{Z=X+Y \in \mathscr{F}(n ; \mathscr{O}) ; \operatorname{det} Y \leq c\}
$$

for sufficiently large $c>1$. Then $\mathscr{C}$ becomes a compact subset with positive volume. Hence the series is majorized by

$$
G_{k}:=\sum_{M \in \mathscr{R}} \int_{M\langle\mathscr{C}\rangle}(\operatorname{det} Y)^{k / 2} d v
$$

in view of Proposition 4.2. Let $l$ denote the number of neighbors of $\mathscr{F}(n ; \mathscr{O})$ and set $\mathscr{U}=\bigcup_{M \in \mathscr{R}} M\langle\mathscr{C}\rangle$. Thus we obtain

$$
G_{k} \leq l \int_{\mathscr{U}}(\operatorname{det} Y)^{k / 2} d v \text {. }
$$

Now $\mathscr{U}$ is contained in a fundamental domain of $\mathscr{H}(n ; \mathbf{F})$ with respect to the action of $\Gamma(n ; \mathscr{O})_{\infty}$. Every $Z=X+Y \in \mathscr{U}$ satisfies det $Y \leq c$ in virtue of $\mathscr{C} \subset \mathscr{F}(n ; \mathscr{O})$. According to (3.3) it suffices to check the convergence of the integral

$$
\int_{\substack{X \in \mathscr{C}(n ; \mathcal{O}), Y \in \mathscr{R}(n ; \mathbf{F}) \\ \operatorname{det} Y \leq c}}(\operatorname{det} Y)^{k / 2} d v .
$$

In view of $d v=2^{r n(n-1) / 2}(\operatorname{det} Y)^{-r n} d X d Y$ it suffices to estimate the integral

$$
\int_{Y \in \mathscr{R}(n ; \mathbf{F}), \operatorname{det} Y \leq c}(\operatorname{det} Y)^{k / 2-r n} d Y .
$$

According to [16], I.5.10, this integral exists, whenever $k>r(n+1)-$ 2.

Thus we can easily derive

THEOREM 4.4. The series

$$
E_{n}^{\mathbf{F}}(Z, s):=\sum_{M: \Gamma_{n}^{\infty} \backslash \Gamma_{n}}\left(\operatorname{det} Y_{M}\right)^{s}
$$

converges absolutely and uniformly, whenever $Z$ belongs to a compact subset of $\mathscr{H}(n ; \mathbf{F})$ and $s \in \mathbf{C}$ satisfies $\operatorname{Re}(s) \geq k, k>\frac{1}{2} r(n+1)-1$. Given $Z \in \mathscr{H}(n ; \mathbf{F})$ the function

$$
\left\{s \in \mathbf{C} ; \operatorname{Re}(s)>\frac{1}{2} r(n+1)-1\right\} \rightarrow \mathbf{C}, \quad s \mapsto E_{n}^{\mathbf{F}}(Z, s),
$$


becomes holomorphic. Let $s \in \mathbf{C}, \operatorname{Re}(s)>\frac{1}{2} r(n+1)-1$, be fixed. Then

$$
E_{n}^{\mathbf{F}}(M\langle Z\rangle, s)=E_{n}^{\mathbf{F}}\left(\bar{Z}^{\prime}, s\right)=E_{n}^{\mathbf{F}}(Z, s)
$$

holds for all $Z \in \mathscr{H}(n ; \mathbf{F})$ and $M \in \Gamma(n ; \mathscr{O})$. Given $\varepsilon>0$ there exists $c>0$ such that

$$
\left|E_{n}^{\mathbf{F}}(Z, s)\right| \leq c(\operatorname{det} Y)^{\operatorname{Re}(s)}
$$

holds for all $Z \in \mathscr{H}(n ; \mathbf{F})$ satisfying $Y \geq \varepsilon I$.

Proof. The definition does not depend on the choice of the representatives in view of (3.3). Using $\operatorname{det} Y_{M}=(\operatorname{det} Y) \cdot|\operatorname{det} M\{Z\}|^{-2}$ the properties of convergence follow from the previous lemma.

The uniform convergence implies that the function $s \mapsto E_{n}^{\mathbf{F}}(Z, s)$ becomes holomorphic. If $K$ then also $K M$, where $M \in \Gamma(n ; \mathscr{O})$, resp. $\tilde{K}$ (cf. Proposition 1.4), run through sets of representatives of $\Gamma_{n}^{\infty} \backslash \Gamma_{n}$. Hence (4.4) follows by a rearrangement. In order to prove (4.5), we may assume $Z \in \mathscr{V} \mathscr{S}_{\varepsilon}(n ; \mathbf{F})$ in virtue of $E_{n}^{\mathbf{F}}(Z+S, s)=E_{n}^{\mathbf{F}}(Z, s)$ for $S \in \operatorname{Alt}(n ; \mathscr{O})$. Then Lemma 4.3 completes the proof.

Definition. $E_{n}^{\mathbf{F}}(Z, s)$ is called Eisenstein-series in $Z$ and $s$.

In virtue of (3.1) the case $\mathbf{F}=\mathbf{R}, n=1$ becomes trivial, namely

$$
E_{1}^{\mathbf{R}}(y, s)=y^{s}+y^{-s}, \quad \text { whenever } y \in \mathscr{H}(1 ; \mathbf{R})=\mathbf{R}^{+} .
$$

Consider the classical non-analytic Eisenstein-series

$$
E(z, s)=\frac{1}{2} \sum_{(c, d) \in \mathbf{Z}^{2} \text { coprime }}\left(\frac{y}{|c z+d|^{2}}\right)^{s},
$$

where $s \in \mathbf{C}, \operatorname{Re}(s)>1, z=x+i y \in \mathbf{C}, y>0$ (cf. [19], [20]). Then (3.2) and [16], II.2.6, imply

$$
E_{1}^{\mathrm{C}}(z, s)=E(i z, s), \quad z \in \mathscr{H}(1 ; \mathbf{C}) .
$$

Consider the Laplace-Beltrami-operator $\Delta$ in Theorem 2.6. Corollary 2.7 immediately leads to

COROllary 4.5. The Eisenstein-series is an eigenfunction of the Laplace-Beltrami-operator. More precisely, if $s \in \mathbf{C}, \operatorname{Re}(s)>\frac{1}{2} r(n+1)$ -1 , then

$$
\Delta E_{n}^{\mathbf{F}}(Z, s)=n s\left(s-\frac{1}{2} r(n+1)+1\right) E_{n}^{\mathbf{F}}(Z, s) .
$$


According to the classical procedure by $\mathrm{H}$. Braun [2], we can show that the abscissa of absolute convergence is given by $\operatorname{Re}(s)=$ $\frac{1}{2} r(n+1)-1$ except for the trivial case (4.6), of course. Therefore some preliminaries are necessary.

A matrix $G \in \operatorname{Mat}(n, m ; \mathscr{O})$, where $m \geq n$ (resp. $n \geq m)$, is called primitive if there exists $U \in \mathrm{GL}(m ; \mathscr{O})$ such that $U=\left(\begin{array}{l}G \\ *\end{array}\right)$ (resp. $U \in$ $\operatorname{GL}(n ; \mathscr{O})$ such that $U=(G, *))$. Clearly if $m \geq n$

$G$ is primitive if and only if $H \in \operatorname{Mat}(m, n ; \mathscr{O})$ exists such that $G H=I$.

In the cases $\mathscr{O}=\mathrm{Z}, \mathrm{Z} e_{1}+\mathrm{Z} e_{2}$ the matrix $G$ proves to be primitive if and only if the $n$-rowed subdeterminants of $G$ are coprime.

Given $M=\left(\begin{array}{ll}A & B \\ C & D\end{array}\right) \in \operatorname{MSp}(n ; \mathbf{F})$ then $(C, D)$ is called the second row of $M$.

Proposition 4.6. The second rows of the matrices in $\Gamma(n ; \mathscr{O})$ coincide with the primitive pairs $(C, D) \in \operatorname{Mat}(n, 2 n ; \mathscr{O})$ satisfying $C \bar{D}^{\prime}+$ $D \bar{C}^{\prime}=0$.

Proof. If $M$ belongs to $\Gamma(n ; \mathscr{O})$, apply $(1.1)$ and use $\Gamma(n ; \mathscr{O}) \subset$ $\mathrm{GL}(2 n ; \mathscr{O})$. Conversely, let such a pair $(C, D)$ be given. According to (4.9) $F, G \in \operatorname{Mat}(n ; \mathscr{O})$ exist such that $C F+D G=I$. Now set

$$
M=\left(\begin{array}{cc}
A & B \\
C & D
\end{array}\right), \quad A:=\bar{G}^{\prime}-\bar{F}^{\prime} G C, \quad B:=\bar{F}^{\prime}-\bar{F}^{\prime} G D
$$

and verify $M \in \Gamma(n ; \mathscr{O})$.

Next we consider $\Gamma(1 ; \mathscr{O}(\mathbf{H}))$ and compute the number of $d$ 's, whenever an odd $c$ is given.

Proposition 4.7. Let $c \in \mathscr{O}(\mathbf{H})$ such that $N(c)$ is odd and set $l:=$ $\max \left\{m \in \mathbf{N} ; \frac{1}{m} c \in \mathscr{O}\right\}$. Then there exist $l \cdot N(c)$ cosets $d+c \operatorname{Alt}(1 ; \mathscr{O})$ such that $c \bar{d}+d \bar{c}=0$.

Proof. We can replace $c$ by $\varepsilon c, \varepsilon \in \mathscr{E}=\{g \in \mathscr{O} ; N(g)=1\}$, and may assume $c=\sum_{j=1}^{4} c_{j} e_{j}, c_{j} \in \mathbf{Z}$. Thus $l=$ g.c.d. $\left(c_{1}, c_{2}, c_{3}, c_{4}\right)$ holds. Let $q=N(c)$, then there are exactly $l q^{3}$ tuples $\left(d_{1}, d_{2}, d_{3}, d_{4}\right)^{\prime}$ in $\mathbf{Z}^{4} \bmod q$ such that

$$
c_{1} d_{1}+c_{2} d_{2}+c_{3} d_{3}+c_{4} d_{4} \equiv 0 \bmod q
$$

holds. Hence there are $l q^{3}$ cosets $d_{j}+q \mathscr{O}$ such that $2 \operatorname{Re}\left(d_{j} \bar{c}\right) \equiv$ 0 mod $q$. Observe that each coset $c \mathscr{O}$ decomposes into $q^{2}$ cosets $d+q \mathscr{O}$ 
(cf. [17]). After renumbering we therefore may assume that

$$
\bigcup_{j=1}^{l q}\left(d_{j}+c \mathscr{O}\right)=\bigcup_{j=1}^{l q^{3}}\left(d_{j}+q \mathscr{O}\right)
$$

Since $q$ is odd, we can choose the representatives such that $\operatorname{Re}\left(d_{j} \bar{c}\right)=0$ holds for $1 \leq j \leq l q$. Hence $d_{j}+c \operatorname{Alt}(1 ; \mathscr{O}), 1 \leq j \leq l q$, are the cosets with the desired property.

Next it is necessary to compute an integral. The same arguments, which were used by H. Braun in [2], [3] resp. in [16], V.1.2, yield

LEMMA 4.8. In the case $\mathbf{F}=\mathbf{R}$ let $n>1, s \in \mathbf{C}, \operatorname{Re}(s)>n-3 / 2$. If $\mathbf{F}=\mathbf{C}, \mathbf{H}$, let $n \geq 1, s \in \mathbf{C}, \operatorname{Re}(s)>r n-1$. Given $Z=X+Y \in \mathscr{H}(n ; \mathbf{F})$ the integral

$$
\eta_{s}(Z):=\int_{\operatorname{Alt}(n ; \mathbf{F})}|\operatorname{det}(Z+T)|^{-s} d T
$$

exists and satisfies

$$
\eta_{s}(Z)=(\operatorname{det} Y)^{r(n+1) / 2-1-s} \eta_{s, n}^{\mathbf{F}}
$$

where

$$
\eta_{s, n}^{\mathbf{F}}=\pi^{r n(n+1) / 4-n / 2} \prod_{j=1}^{n} \frac{\Gamma\left(s+1-\frac{1}{2} r(n+j)\right)}{\Gamma(s+1-r j)} \frac{\Gamma\left(\frac{1}{2}(s+1-r j)\right)}{\Gamma\left(\frac{1}{2}(s+r-r j)\right)}
$$

Note that in the case $\mathbf{F}=\mathbf{R}$, i.e. $r=1$, several factors on the righthand side can be reduced such that the reduced product even exists for $\operatorname{Re}(s)>n-3 / 2$. Here $\Gamma(s)$ denotes the gamma-function, since confusion with the modular group is not possible.

The existence of the integral implies the convergence of a series.

Corollary 4.9. Let $k \in \mathbf{R}$ and $k>n-3 / 2, n>1$ for $\mathbf{F}=\mathbf{R}$ resp. $k>r n-1, n \geq 1$ for $\mathbf{F}=\mathbf{C}, \mathbf{H}$. Given $\varepsilon>0$ there exists $c>0$ such that

$$
c^{-k} \eta_{k}(Z) \leq \sum_{T \in \operatorname{Alt}(n ; \mathcal{O})}|\operatorname{det}(Z+T)|^{-k} \leq c^{k} \eta_{k}(Z)
$$

holds for all $Z=X+Y \in \mathscr{H}(n ; \mathbf{F})$ satisfying $Y \geq \varepsilon I$.

Proof. The assertion follows from an estimation between $|\operatorname{det}(Z+T)|^{-k}$ and

$$
\int_{\mathscr{C}(n ; \mathcal{O})}|\operatorname{det}(Z+T+H)|^{-k} d H
$$


This estimation can be derived by (1.10), (1.11), (1.12) and [16], V.1.4.

Now we follow $\mathrm{H}$. Braun [2] in order to determine the abscissa of convergence of the Eisenstein-series. Hereby the result on real Eisenstein-series quoted by $\mathrm{H}$. Maaß [23] can even be strengthened.

THEOREM 4.10. Let $n>1$ for $\mathbf{F}=\mathbf{R}$ and $n \geq 1$ for $\mathbf{F}=\mathbf{C}, \mathbf{H}$. Then the Eisenstein-series $E_{n}^{\mathbf{F}}(Z, s)$ does not converge absolutely, whenever $\operatorname{Re}(s)=\frac{1}{2} r(n+1)-1$.

Proof. According to Proposition 4.2 it suffices to show that the series

$$
E_{n}^{\mathbf{F}}(I, k)=\sum_{M: \Gamma_{n}^{\infty} \backslash \Gamma_{n}}|\operatorname{det} M\{I\}|^{-2 k}, \quad k=\frac{1}{2} r(n+1)-1,
$$

diverges. Therefore we take second rows $(C, D)$ of matrices $M \in$ $\Gamma(n ; \mathscr{O})$ such that the cosets $\Gamma_{n}^{\infty} M\left(\begin{array}{ll}I & S \\ 0 & I\end{array}\right), S \in \operatorname{Alt}(n ; \mathscr{O})$, are mutually disjoint. In view of

$$
\begin{aligned}
E_{n}^{\mathbf{F}}(I, k) & \geq \sum_{M\left(\begin{array}{l}
I S \\
0
\end{array}\right)}|\operatorname{det} M\{I\}|^{-2 k} \\
& =\sum_{C, D, S}|\operatorname{det} C|^{-2 k}\left|\operatorname{det}\left(I+C^{-1} D+S\right)\right|^{-2 k}
\end{aligned}
$$

and Corollary 4.9 it suffices to estimate

$$
E_{k}:=\sum_{C, D}|\operatorname{det} C|^{-2 k}
$$

In the case $\mathbf{F}=\mathbf{R}, n \geq 2$ choose

$$
C=\left(\begin{array}{cc}
c I^{(2)} & 0 \\
G & I
\end{array}\right), \quad D=\left(\begin{array}{cc}
d J & -d J G^{\prime} \\
0 & 0
\end{array}\right), \quad J=\left(\begin{array}{cc}
0 & 1 \\
-1 & 0
\end{array}\right),
$$

where $c \in \mathbf{N}, d, 1 \leq d \leq c$, is relatively prime to $c$ and $G$ runs through a set of representatives of $\operatorname{Mat}(n-2,2 ; \mathbf{Z}) / c \operatorname{Mat}(n-2,2 ; \mathbf{Z})$, which consists of $c^{2 n-4}$ elements. $(C, D)$ has the desired property. If $\varphi$ denotes Euler's $\varphi$-function, we obtain $k=\frac{1}{2}(n-1)$ and

$$
E_{k}=\sum_{c, d} c^{-2}=\sum_{c=1}^{\infty} \varphi(c) c^{-2}
$$

But this series diverges.

In the case $\mathbf{F}=\mathbf{C}$ apply [3], Theorem II. 
In the case $\mathbf{F}=\mathbf{H}$ let $c$ run through a system of representatives of

$$
\mathscr{E} \backslash\{x \in \mathscr{O} ; N(x)=p\},
$$

where $\mathscr{E}=\{g \in \mathscr{O} ; N(g)=1\}$ and $p$ runs through all odd primes. For every prime $p$ we have $p+1$ possibilities for $c$ according to [9]. Given $c$ choose $d_{1}, \ldots, d_{p}$ according to Proposition 4.7 and assume $d_{p}=0$. Hence we may suppose $p \nmid N\left(d_{j}\right)$ for $1 \leq j<p$. Set $x=\left(c_{2}, \ldots, c_{n}\right)^{\prime}$ and let each $c_{j}$ run through a set of representatives of $\mathscr{O} / \mathscr{O} c$, which consists of $N(c)^{2}=p^{2}$ elements (cf. [17]). Now set

$$
C=\left(\begin{array}{cc}
c & 0 \\
x & I
\end{array}\right), \quad D=\left(\begin{array}{cc}
d & -d \bar{x}^{\prime} \\
0 & 0
\end{array}\right), \quad d=d_{j}, 1 \leq j<p,
$$

and observe that $(C, D)$ has the desired property. Now we obtain $k=2 n+1$ and

$$
E_{k}=\sum_{p>2 \text { prime }}(p-1)(p+1) p^{-3}
$$

This series diverges.

Just as in the case of Siegel modular forms we can define a modified $\phi$-operator. Given a function $f: \mathscr{H}(n ; \mathbf{F}) \rightarrow \mathbf{C}$ and $s \in \mathbf{C}$, we set

$$
\left.f\right|_{s} \phi: \mathscr{H}(n-1 ; \mathbf{F}) \rightarrow \mathbf{C}, \quad Z \mapsto \lim _{\lambda \rightarrow \infty} \lambda^{-s} f\left(\left(\begin{array}{cc}
Z & 0 \\
0 & \lambda
\end{array}\right)\right),
$$

if this limit exists. $f \mid{ }_{s} \phi$ has to be regarded as a constant, if $n=1$. Then $\phi$ is called the modified Siegel $\phi$-operator.

Finally we show that the modified Siegel $\phi$-operator can be applied to Eisenstein-series just as in the classical case.

THEOREM 4.11. Given $s \in \mathbf{C}, \operatorname{Re}(s)>\frac{1}{2} r(n+1)-1$, then one has

$$
\begin{aligned}
& \left.E_{n}^{\mathbf{F}}(\cdot, s)\right|_{s} \phi=E_{n-1}^{\mathbf{F}}(\cdot, s) \text { for } n \geq 2, \\
& E_{1}^{\mathbf{F}}(\cdot, s){ }_{s} \phi=1 .
\end{aligned}
$$

Proof. According to Lemma 4.3 the limit may be distributed through the infinite series. The case $n=1$ becomes clear in view of

$$
\lim _{\lambda \rightarrow \infty}|M\{\lambda\}|^{-2}=\lim _{\lambda \rightarrow \infty} N(c \lambda+d)^{-1}= \begin{cases}N(d)^{-1} & \text { if } c=0, \\ 0 & \text { if } c \neq 0 .\end{cases}
$$

Let $n \geq 2$ and let $\Gamma_{n}^{*}$ denote the set of matrices $M \in \Gamma_{n}$ such that the elements $m_{2 n, j}, 1 \leq j<2 n$, vanish. $\Gamma_{n}^{*}$ proves to be a subgroup and one easily verifies that the map

$$
\Gamma_{n-1}^{\infty} \backslash \Gamma_{n-1} \rightarrow\left(\Gamma_{n}^{*} \cap \Gamma_{n}^{\infty}\right) \backslash \Gamma_{n}^{*}, \quad \Gamma_{n-1}^{\infty} M \mapsto\left(\Gamma_{n}^{*} \cap \Gamma_{n}^{\infty}\right)\left(M \times I^{(2)}\right),
$$


becomes a bijection. Let $Z_{\lambda}:=\left(\begin{array}{cc}Z & 0 \\ 0 & \lambda\end{array}\right)$. Given $M \in \Gamma_{n}^{*}$ then $\left|\operatorname{det} M\left\{Z_{\lambda}\right\}\right|$ does not depend on $\lambda$. Hence we obtain

$$
\sum_{M:\left(\Gamma_{n}^{*} \cap \Gamma_{n}^{\infty}\right) \backslash \Gamma_{n}^{*}}(\operatorname{det} Y)^{s}\left|\operatorname{det} M\left\{Z_{\lambda}\right\}\right|^{-2 s}=E_{n-1}^{\mathbf{F}}(Z, s) .
$$

Given $M \in \Gamma(n ; \mathscr{O})$ such that $\Gamma_{n}^{\infty} M \cap \Gamma_{n}^{*}=\varnothing$ one checks that $\lim _{\lambda \rightarrow \infty}\left|M\left\{Z_{\lambda}\right\}\right|=\infty$ holds.

The isomorphisms $\chi_{2}$ and $\chi_{3}$ in Remark 2.3 between symmetric spaces correspond to identities between the associated Eisensteinseries. Therefore the Eisenstein-series (4.7) and Eisenstein-series for $\mathrm{GL}(4 ; \mathbf{Z})$, which were investigated by A. Terras [31], appear. Note that the action of $\Gamma(3 ; \mathbf{Z})_{\infty}$ corresponds to the action of the parabolic subgroup $P_{3,1}$ of $\mathrm{GL}(4 ; \mathbf{Z})$ via $\chi_{3}$. Consider the attached Eisenstein-series of the second type in [31]

$$
E_{s, 0}(Y):=\sum_{P: \operatorname{Pr}(4,3, \mathbf{Z}) / \mathrm{GL}(3 ; \mathbf{Z})}(\operatorname{det} Y[P])^{-s},
$$

where $Y \in \operatorname{SPos}(4 ; \mathbf{R})$ and $\operatorname{Pr}(4,3, Z)$ denotes the set of primitive $4 \times 3$ matrices over $\mathbf{Z}$. Thus an explicit computation yields

LEMMA 4.12. (a) Given

$$
Z=x J+Y=\left(\begin{array}{ll}
y_{1} & y+x \\
y-x & y_{2}
\end{array}\right) \in \mathscr{H}(2 ; \mathbf{R})
$$

and $s \in \mathrm{C}$ with $\operatorname{Re}(s)>\frac{1}{2}$ one has

$$
E_{2}^{\mathbf{R}}(Z, s)=E(x+i \sqrt{\operatorname{det} Y}, 2 s)+E\left(\frac{1}{y_{1}}(-y+i \sqrt{\operatorname{det} Y}), 2 s\right) .
$$

(b) Given $Z \in \mathscr{H}(3 ; \mathbf{R})$ and $s \in \mathbf{C}$ with $\operatorname{Re}(s)>1$ one has

$$
E_{3}^{\mathbf{R}}(Z, s)=E_{2 s, 0}\left(\chi_{3}(Z)\right)+E_{2 s, o}\left(\chi_{3}(Z)^{-1}\right) .
$$

5. Fourier-expansion of Eisenstein-series. The Fourier-expansion of non-analytic Eisenstein-series on the Siegel half-space was investigated by H. Maaß [22], §18. G. Shimura [27] dealt with the case $\mathbf{F}=\mathbf{C}$, if we regard (0.2) and (1.9). Some of the following results on real Eisenstein-series were already obtained by $\mathrm{H}$. Maaß [23].

Throughout this paragraph let $s \in \mathbf{C}$ be fixed such that $\operatorname{Re}(s)>$ $\frac{1}{2} r(n+1)-1$ holds. In order to describe the Fourier-development, we have to determine the dual lattice. Therefore set

$$
\begin{aligned}
& \mathscr{O}^{\#}(\mathbf{F})=\mathscr{O}(\mathbf{F}), \quad \mathbf{F}=\mathbf{R}, \mathbf{C}, \\
& \mathscr{O}^{\#}(\mathbf{H})=\mathbf{Z} 2 e_{1}+\mathbf{Z}\left(e_{1}+e_{2}\right)+\mathbf{Z}\left(e_{1}+e_{3}\right)+\mathbf{Z}\left(e_{1}+e_{4}\right)
\end{aligned}
$$


(cf. [16], p. 12). Using the definition of $\tau$ in $\S 2$ we derive

$$
\begin{aligned}
\operatorname{Alt}^{\tau}(n ; \mathscr{O}) & :=\{T \in \operatorname{Alt}(n ; \mathbf{F}) ; \tau(T, S) \in \mathbf{Z} \text { for all } S \in \operatorname{Alt}(n ; \mathscr{O})\} \\
& =\left\{T=\left(t_{k l}\right) \in \operatorname{Alt}(n ; \mathbf{F}) ; t_{k k} \in \mathscr{O}, 2 t_{k l} \in \mathscr{O}^{\#} \text { for } k \neq l\right\} .
\end{aligned}
$$

Since the Eisenstein-series is invariant under the transformations $Z \mapsto Z+S, S \in \operatorname{Alt}(n ; \mathscr{O})$, we obtain

$$
E_{n}^{\mathbf{F}}(Z, s)=\sum_{T \in \mathrm{Alt}^{\tau}(n ; \mathcal{O})} c(Y ; T) e^{2 \pi i \tau(X, T)}, \quad Z=X+Y \in \mathscr{H}(n ; \mathbf{F}) .
$$

The use of $E_{n}^{\mathbf{F}}(Z[U], s)=E_{n}^{\mathbf{F}}\left(\bar{Z}^{\prime}, s\right)=E_{n}^{\mathbf{F}}(Z, s)$ according to (4.4) as well as the uniqueness of the Fourier-coefficients yield

$$
c(Y[U] ; T)=c\left(Y ; T\left[\bar{U}^{\prime}\right]\right), \quad c(Y ; T)=c(Y ;-T)
$$

for all $U \in \mathrm{GL}(n ; \mathscr{O})$.

It is convenient to decompose the Eisenstein-series into $n+1$ partial series. Given $0 \leq j \leq n$ we set

$$
E_{n, j}^{\mathbf{F}}(Z, s)=\sum_{\substack{M: \Gamma_{n}^{\infty} \backslash \Gamma_{n} \\ \operatorname{rank} C=j}}\left(\operatorname{det} Y_{M}\right)^{s}
$$

The definition leads to the obvious relations

$$
\begin{gathered}
E_{n}^{\mathbf{F}}(Z, s)=\sum_{j=0}^{n} E_{n, j}^{\mathbf{F}}(Z, s), \\
E_{n, 0}^{\mathbf{F}}(Z, s)=(\operatorname{det} Y)^{s} .
\end{gathered}
$$

Set $\operatorname{Pr}(n, m ; \mathscr{O}):=\{G \in \operatorname{Mat}(n, m ; \mathscr{O}) ; G$ primitive $\}$. Following H. Maaß [22], $\S 11$, the same arguments yield

Lemma 5.1. Given $0<j<n$ let $P$ run through a set of representatives of $\operatorname{Pr}(n, j ; \mathscr{O}) / \mathrm{GL}(j ; \mathscr{O})$. Each $P$ is completed to a matrix $U=(P, *) \in \mathrm{GL}(n ; \mathscr{O})$ in exactly one way. Let $M_{1}$ run through the subset of representatives of $\Gamma_{j}^{\infty} \backslash \Gamma_{j}$, where $\left|\operatorname{det} C_{1}\right| \neq 0$. Then $\left(M_{1} \times I\right)\left(\begin{array}{cc}\bar{U}^{\prime} & 0 \\ 0 & U^{-1}\end{array}\right)$ runs through the subset of representatives of $\Gamma_{n}^{\infty} \backslash \Gamma_{n}$, where $\operatorname{rank} C=j$.

Thus we easily compute

Corollary 5.2. Given $0<j<n$ one has

$$
E_{n, j}^{\mathrm{F}}(Z, s)=\sum_{P: \operatorname{Pr}(n, j ; \mathcal{O}) / \mathrm{GL}(j ; \mathcal{O})}(\operatorname{det} Y)^{s}(\operatorname{det} Y[P])^{-s} E_{j, j}^{\mathbf{F}}(Z[P], s) .
$$


Given $S \in \operatorname{Pos}(n ; \mathbf{R}), 0<j<n$, and $\omega \in \mathbf{C}$ satisfying $\operatorname{Re}(\omega)>\frac{1}{2} n$, we can define the Dirichlet-series

$$
\zeta_{j}(S, \omega):=\sum_{P: \operatorname{Pr}(n, j ; \mathbf{Z}) / \mathrm{GL}(j ; \mathbf{Z})}(\operatorname{det} S[P])^{-\omega} .
$$

A related series was investigated by $M$. Koecher [13]. $\zeta_{1}(S, \omega)$ proves to be the quotient of the corresponding Epstein-zeta-function over the Riemann-zeta-function $2 \zeta(2 \omega)$. In view of $(5.1),(5.2),(4.6)$ and Corollary 5.2 we gain

$$
E_{n, 1}^{\mathbf{R}}(Z, s)=(\operatorname{det} Y)^{s} \zeta_{1}(Y, 2 s),
$$

whenever $n \geq 2$.

In view of the corollary the problem is reduced to the investigation of $E_{n, n}^{\mathbf{F}}(Z, s)$. Set $\mathbf{F}_{\mathbf{Q}}=\mathbf{Q} e_{1}+\cdots+\mathbf{Q} e_{r}$. The matrices in $\operatorname{Mat}\left(n ; \mathbf{F}_{\mathbf{Q}}\right)$ are called rational.

LEMMA 5.3. Let $M=\left(\begin{array}{ll}A & B \\ C & D\end{array}\right)$ run through the subset of representatives of $\Gamma_{n}^{\infty} \backslash \Gamma_{n}$, where rank $C=n$. Then each $R \in \operatorname{Alt}\left(n ; \mathbf{F}_{\mathbf{Q}}\right)$ is represented in the form $R=C^{-1} D$ exactly once. Moreover

$$
\nu(R)=|\operatorname{det} C|
$$

becomes well-defined and satisfies

$$
\nu(R+S)=\nu(R) \quad \text { for } S \in \operatorname{Alt}(n ; \mathscr{O}) .
$$

If $\mathscr{O}=\mathbf{Z}, \mathbf{Z} e_{1}+\mathbf{Z} e_{2}$, then $\nu(R)$ coincides with the absolute value of the product of the denominators of the reduced elementary divisors of $R$.

Proof. Given $R \in \operatorname{Alt}\left(n ; \mathbf{F}_{\mathbf{Q}}\right)$ choose $U, V \in \mathrm{GL}(n ; \mathscr{O})$ such that

$$
U R V=\left[q_{1}, \ldots, q_{n}\right], \quad q_{j} \in \mathbf{F}_{\mathbf{Q}}, \quad q_{j+1} \in \mathscr{O} q_{j},
$$

according to [16], 1.2.3. Each $q_{j}$ possesses a representation $q_{j}=$ $c_{j}^{-1} d_{j}, c_{j} \neq 0, c_{j}, d_{j} \in \mathscr{O}$, where $c_{j}$ and $d_{j}$ are relatively left-prime. Define $C_{0}=\left[c_{1}, \ldots, c_{n}\right], D_{0}=\left[d_{1}, \ldots, d_{n}\right]$, then $\left(C_{0}, D_{0}\right)$ becomes primitive (cf. [16], I.1.11). Hence $(C, D):=\left(C_{0} U, D_{0} V^{-1}\right)$ proves to be primitive and satisfies $\operatorname{rank} C=n$ as well as

$$
C^{-1} D=U^{-1}\left[q_{1}, \ldots, q_{n}\right] V^{-1}=R .
$$

Now $(C, D)$ turns out to be the second row of a matrix in $\Gamma(n ; \mathscr{O})$ according to Proposition 4.6. If $\mathscr{O}=\mathrm{Z}, \mathrm{Z} e_{1}+\mathrm{Z} e_{2}$, moreover $|\operatorname{det} C|$ equals the absolute value of the product of the denominators of the reduced elementary divisors of $R$. 
Clearly, the representation $R=C^{-1} D$ and $|\operatorname{det} C|$ do not depend on the choice of the representative in the coset $\Gamma_{n}^{\infty} M$ in view of (3.3). Now suppose that $M=\left(\begin{array}{ll}A & B \\ C & D\end{array}\right)$ and $M_{1}=\left(\begin{array}{ll}A_{1} & B_{1} \\ C_{1} & D_{1}\end{array}\right)$ belong to $\Gamma(n ; \mathscr{O})$ and fulfill rank $C=\operatorname{rank} C_{1}=n$ as well as $C^{-1} D=C_{1}^{-1} D_{1}=R$. Then $\bar{R}^{\prime}=-R$ yields $C \bar{D}_{1}^{\prime}+D \bar{C}_{1}^{\prime}=0$. Hence (1.2) implies $M M_{1}^{-1} \in \Gamma_{n}^{\infty}$, i.e. $\Gamma_{n}^{\infty} M=\Gamma_{n}^{\infty} M_{1}$. Replacing $M$ by $M\left(\begin{array}{ll}I & S \\ 0 & I\end{array}\right), S \in \operatorname{Alt}(n ; \mathscr{O})$, yields $\nu(R+S)=\nu(R)$.

In the case $\mathscr{O}=\mathrm{Z}$ we obtain information about the elementary divisor normal form of the $C$-block in a matrix $M \in \Gamma(n ; \mathbf{Z})$.

Corollary 5.4. Given $M=\left(\begin{array}{ll}A & B \\ C & D\end{array}\right) \in \Gamma(n ; Z)$ then the elementary divisor matrix of $C$ has the form

$$
\begin{array}{ll}
{\left[c_{1}, c_{1}, c_{2}, c_{2}, \ldots, c_{m}, c_{m}, 0, \ldots, 0\right],} & \text { if } \operatorname{rank} C=2 m, \\
{\left[1, c_{1}, c_{1}, c_{2}, c_{2}, \ldots, c_{m}, c_{m}, 0, \ldots, 0\right],} & \text { if } \operatorname{rank} C=2 m+1,
\end{array}
$$

where $c_{1}, \ldots, c_{m} \in \mathbf{N}$ such that $c_{j} \mid c_{j+1}$.

Proof. We may assume $\operatorname{rank} C=n$. Then a combination of [25], Theorem IV.1, with Lemma 5.3 yields the assertion.

Replacing $M$ by a product of $M$ and $Q$ a corresponding result is true for each other block of the matrix $M \in \Gamma(n ; \mathbf{Z})$.

Furthermore, Lemma 5.3 immediately yields

$$
E_{n, n}^{\mathbf{F}}(Z, s)=(\operatorname{det} Y)^{s} \sum_{R \in \operatorname{Alt}\left(n ; \mathbf{F}_{\mathbf{Q}}\right)} \nu(R)^{-2 s}|\operatorname{det}(Z+R)|^{-2 s} .
$$

In view of $\nu(R+S)=\nu(R)$ for $S \in \operatorname{Alt}(n ; \mathscr{O})$, the partial series $E_{n, j}^{\mathbf{F}}(Z, s)$ possesses a Fourier-expansion, too. Let $R \bmod 1$ indicate that $R$ runs through a set of representatives of $\operatorname{Alt}\left(n ; \mathbf{F}_{\mathbf{Q}}\right) / \operatorname{Alt}(n ; \mathscr{O})$. Given $T \in \operatorname{Alt}^{\tau}(n ; \mathscr{O})$ and $Y \in \operatorname{Pos}(n ; \mathbf{F})$, we define

$$
\begin{aligned}
\alpha_{s}(T) & :=\sum_{R \bmod 1} \nu(R)^{-2 s} e^{2 \pi i \tau(R, T)}, \\
\beta_{s}(Y ; T) & :=\int_{\operatorname{Alt}(n ; \mathbf{F})}|\operatorname{det}(Y+X)|^{-2 s} e^{-2 \pi i \tau(X, T)} d X .
\end{aligned}
$$

Given $U \in \mathrm{GL}(n ; \mathscr{O})$ we immediately obtain

$$
\begin{aligned}
\alpha_{s}(T[U]) & =\alpha_{s}(-T)=\alpha_{s}(T), \\
\beta_{s}(Y ; T[U]) & =\beta_{s}\left(Y\left[\bar{U}^{\prime}\right] ; T\right), \quad \beta_{s}(Y ; T)=\beta_{s}(Y ;-T) .
\end{aligned}
$$

Hence Lemma 5.3 and the definition of the Fourier-coefficients imply 
LEMMA 5.5.

$$
E_{n, n}^{\mathbf{F}}(Z, s)=(\operatorname{vol} \mathscr{C}(n ; \mathscr{O}))^{-1} \sum_{T \in \mathrm{Alt}^{\tau}(n ; \mathcal{O})}(\operatorname{det} Y)^{s} \alpha_{s}(T) \beta_{s}(Y ; T) e^{2 \pi i \tau(X, T)}
$$

Combining this result with (5.1) and Corollary 5.2, we gain

Corollary 5.6.

$$
\begin{aligned}
E_{n}^{\mathbf{F}}(Z, s)= & (\operatorname{det} Y)^{s}+(\operatorname{det} Y)^{s} \\
& \times \sum_{j=1}^{n} c_{j}^{-1} \sum_{P} \sum_{T \in \operatorname{Alt}^{\tau}(j ; \mathscr{O})} \alpha_{s}(T) \beta_{s}(Y[P] ; T) e^{2 \pi i \tau\left(X, T\left[\bar{P}^{\prime}\right]\right)},
\end{aligned}
$$

where $c_{j}=\operatorname{vol} \mathscr{C}(j ; \mathscr{O})$ and $P: \operatorname{Pr}(n, j ; \mathscr{O}) / \mathrm{GL}(j ; \mathscr{O})$.

As a consequence we observe that in the Fourier-expansion of $E_{n, j}^{\mathbf{F}}(Z, s)$ all the coefficients of matrices $T \in \operatorname{Alt}^{\tau}(n ; \mathscr{O})$ vanish, whenever $\operatorname{rank} T>j$.

Lemma 4.8 yields

$$
\beta_{s}(Y ; 0)=(\operatorname{det} Y)^{r(n+1) / 2-1-2 s} \eta_{2 s, n}^{\mathbf{F}} .
$$

REMARK 5.7. It is possible to reduce the computation of $\beta_{S}(Y ; T)$ to the case $|\operatorname{det} T| \neq 0$ by aid of (5.5). Therefore let

$$
\begin{aligned}
T & =\left(\begin{array}{ll}
T_{1} & 0 \\
0 & 0
\end{array}\right) \in \operatorname{Alt}^{\tau}(n ; \mathscr{O}), \quad Y=\left(\begin{array}{cc}
Y_{1} & * \\
* & *
\end{array}\right) \in \operatorname{Pos}(n ; \mathbf{F}), \\
T_{1} & =T_{1}^{(m)}, \quad Y_{1}=Y_{1}^{(m)} .
\end{aligned}
$$

Then one obtains

$$
\begin{aligned}
\beta_{s}(Y ; T)= & \beta_{s-r(n-m) / 2}\left(Y_{1} ; T_{1}\right)(\operatorname{det} Y)^{r(n+1) / 2-2 s} \\
& \cdot\left(\operatorname{det} Y_{1}\right)^{2 s+1+r(m-1-2 n) / 2} \eta_{2 s, n-m}^{\mathbf{F}} \pi^{r m(n-m) / 2} \\
& \cdot \prod_{j=1}^{n-m} \frac{\Gamma\left(2 s+1-\frac{1}{2} r(n+j)\right)}{\Gamma\left(2 s+1-\frac{1}{2} r(n-m+j)\right)} .
\end{aligned}
$$

In general the evaluation of the integral $\beta_{S}(Y ; T)$ leads to generalized confluent hypergeometric functions, where the case $\mathbf{F}=\mathbf{C}$ was treated by G. Shimura [26]. On the other hand it might be possible to investigate $\alpha_{s}(T)$ in analogy with Y. Kitaoka's procedure [11] in the case of the Siegel half-space. But it seems to be plausible that the Fourier-coefficients of the Eisenstein-series can only be expressed by well-known functions, whenever the degree $n$ is "sufficiently small". 
Therefore let us consider the case $n=1$. Now $\mathbf{F}=\mathbf{R}$ becomes trivial in view of (4.6). Dealing with $\mathbf{F}=\mathbf{C}$ we observe the connection (4.8) with the classical Eisenstein-series and obtain the Fourier-expansion from [19], p. 46, or [20].

In order to deal with the case $\mathbf{F}=\mathbf{H}$, it is more convenient to introduce the subring $\Lambda:=\mathbf{Z} e_{1}+\mathbf{Z} e_{2}+\mathbf{Z} e_{3}+\mathbf{Z} e_{4}$ of $\mathscr{O}(\mathbf{H})$. Given $0 \neq c \in \Lambda$ define the greatest rational divisor of $c$ in $\Lambda$ by

$$
\rho(c):=\max \left\{l \in \mathbf{N} ; l^{-1} c \in \Lambda\right\}
$$

and set $\rho(0):=0$. Note that $\operatorname{Alt}(1 ; \mathscr{O})=\operatorname{Alt}^{\tau}(1 ; \mathscr{O})=\mathbf{Z} e_{2}+\mathbf{Z} e_{3}+\mathbf{Z} e_{4} \subset$ $\Lambda$.

Given $S \in \operatorname{Pos}(n ; \mathbf{R})$ and $s \in \mathbf{C}$ with $\operatorname{Re}(s)>\frac{1}{2} n$, the Epstein-zetafunction associated with $S$ is defined by

$$
\zeta(S ; s):=\sum_{0 \neq g \in \mathbf{Z}^{n}}(S[g])^{-s} \text {. }
$$

Especially one has for $I=I^{(4)}$ and $s \in \mathrm{C}$ with $\operatorname{Re}(s)>2$

$$
\zeta(I ; s)=\sum_{0 \neq c \in \Lambda} N(c)^{-s}=8\left(1-2^{2-2 s}\right) \zeta(s) \zeta(s-1),
$$

where $\zeta$ denotes the Riemann-zeta-function. Given $t, t^{*} \in \operatorname{Alt}(1 ; \mathscr{O})$ the Fourier-expansion involves the function

$$
\sigma_{s}\left(t, t^{*}\right):=\sum_{\substack{0 \neq c \in \Lambda \\ c t=t^{*} c}} N(c)^{-s} .
$$

Clearly $\sigma_{s}\left(t, t^{*}\right)=0$ unless $N(t)=N\left(t^{*}\right)$. The structure of $\sigma_{s}\left(t, t^{*}\right)$ is elucidated by

Proposition 5.8. Let $t, t^{*} \in \operatorname{Alt}(1 ; \mathscr{O})$ with $N(t)=N\left(t^{*}\right) \neq 0$ and $s \in \mathbf{C}$ with $\operatorname{Re}(s)>1$. Then there exists $S \in \operatorname{Pos}(2 ; \mathbf{Z})$ such that

$$
\sigma_{s}\left(t, t^{*}\right)=\zeta(S ; s) \quad \text { and } \quad \operatorname{det} S=\frac{4 N(t)}{\left[\operatorname{gcd}\left(\rho\left(t+t^{*}\right), \rho\left(t-t^{*}\right)\right)\right]^{2}}
$$

Proof. Let

$$
t=\sum_{j=2}^{4} t_{j} e_{j}, \quad t^{*}=\sum_{j=2}^{4} t_{j}^{*} e_{j} .
$$

Then $c=\sum_{j=1}^{4} c_{j} e_{j}$ satisfies $c t=t^{*} c$ if and only if $\left(c_{1}, c_{2}, c_{3}, c_{4}\right)^{\prime}$ belongs to the kernel of the matrix

$$
\left(\begin{array}{cccc}
t_{2}-t_{2}^{*} & 0 & t_{4}+t_{4}^{*} & -t_{3}-t_{3}^{*} \\
0 & t_{2}-t_{2}^{*} & t_{3}-t_{3}^{*} & t_{4}-t_{4}^{*} \\
t_{4}-t_{4}^{*} & t_{3}+t_{3}^{*} & -t_{2}-t_{2}^{*} & 0 \\
-t_{3}+t_{3}^{*} & t_{4}+t_{4}^{*} & 0 & -t_{2}-t_{2}^{*}
\end{array}\right)
$$


which has the rank 2 . Hence $\sigma_{s}(t, t)=\zeta(S ; s)$ holds for an appropriate $S \in \operatorname{Pos}(2 ; \mathbf{Z})$. If $t_{2} \neq t_{2}^{*}$ the kernel over $\mathbf{Q}$ is spanned by $a=$ $\left(t_{4}+t_{4}^{*}, t_{3}-t_{3}^{*},-t_{2}+t_{2}^{*}, 0\right)^{\prime}$ and $b=\left(t_{3}+t_{3}^{*},-t_{4}+t_{4}^{*}, 0, t_{2}-t_{2}^{*}\right)^{\prime}$. Hence we have

$$
\operatorname{det} S=\frac{\operatorname{det}\left(G^{\prime} G\right)}{\left[\delta_{2}(G)\right]^{2}}, \quad G=(a, b) \in \operatorname{Mat}(4,2 ; \mathbf{Z}),
$$

where $\delta_{2}(G)$ denotes the second determinantal divisor of $G$ (cf. [25], p. 25). An elementary computation yields $\operatorname{det}\left(G^{\prime} G\right)=4\left(t_{2}-t_{2}^{*}\right)^{2} N(t)$ and $\delta_{2}(G)=\left(t_{2}-t_{2}^{*}\right) \operatorname{gcd}\left(\rho\left(t+t^{*}\right), \rho\left(t-t^{*}\right)\right)$. In the case $t_{2}=t_{2}^{*}$ analogous arguments complete the proof.

If $K_{s}$ denotes the modified Bessel-function, the Fourier-expansion is given by

THEOREM 5.9.

$$
E_{1}^{\mathbf{H}}(z, s)=\sum_{t \in \mathbf{Z} e_{2}+\mathbf{Z} e_{3}+\mathbf{Z} e_{4}} c(y ; t) e^{2 \pi i \operatorname{Re}(\bar{x} t)},
$$

where $z=x+y \in \mathscr{H}(1 ; \mathbf{H})$ and with $I=I^{(4)}$

$$
\begin{aligned}
c(y ; 0)= & y^{s}+\pi^{3 / 2} \frac{\Gamma(s-3 / 2) \zeta(I ; s-1) \zeta(2 s-3)}{\Gamma(s) \zeta(I ; s) \zeta(2 s-2)} y^{3-s}, \\
c(y ; t)= & 2 \pi^{s} \frac{\sum_{l \mid \rho(t)} l^{3-2 s} \sum_{t^{*} \in \operatorname{Alt}(1 ; \mathcal{O})} \sigma_{s-1}\left(t, t+2 l t^{*}\right)}{\Gamma(s) \zeta(I ; s) \zeta(2 s-2)} \\
& \cdot|t|^{s-3 / 2} y^{3 / 2} K_{s-3 / 2}(2 \pi|t| y)
\end{aligned}
$$

for $0 \neq t \in \mathbf{Z} e_{2}+\mathbf{Z} e_{3}+\mathbf{Z} e_{4}$.

Proof. At first (5.6) yields

$$
\beta_{s}(y ; 0)=\pi^{3 / 2} \frac{\Gamma(s-3 / 2)}{\Gamma(s)} y^{3-2 s} .
$$

Given $0 \neq t \in \mathbf{Z} e_{2}+\mathbf{Z} e_{3}+\mathbf{Z} e_{4}$ we use an orthogonal transformation and apply [24], p. 85, in the following calculation

$$
\begin{aligned}
& \beta_{s}(y ; t)=\int_{\mathrm{Alt}(1 ; \mathbf{H})}|y+x|^{-2 s} e^{-2 \pi i \operatorname{Re}(\bar{x} t)} d x \\
& =y^{3-2 s} \int_{-\infty}^{+\infty} \int_{-\infty}^{+\infty} \int_{-\infty}^{+\infty}\left(1+x_{1}^{2}+x_{2}^{2}+x_{3}^{2}\right)^{-s} e^{-2 \pi i y|t| x_{1}} d x_{1} d x_{2} d x_{3} \\
& \quad=2 \pi^{s} \frac{1}{\Gamma(s)} y^{3 / 2-s}|t|^{s-3 / 2} K_{s-3 / 2}(2 \pi|t| y) .
\end{aligned}
$$


Next observe that the representatives of $\Gamma_{1}^{\infty} \backslash \Gamma_{1}$ may be chosen in $\operatorname{Mat}(2 ; \Lambda)$. Given $0 \neq c \in \Lambda$ let $\mathscr{R}(c)$ denote a set of representatives of the cosets $d+c \operatorname{Alt}(1 ; \mathscr{O}), d \in \Lambda$, satisfying $c \bar{d}+d \bar{c}=0$. In analogy with Proposition 4.7 one can show that $\mathscr{R}(c)$ consists of $\rho(c) N(c)$ elements. Moreover we use the abbreviation

$$
\gamma(c, t):=\sum_{d \in \mathscr{R}(c)} e^{2 \pi i \operatorname{Re}\left(c^{-1} d \bar{t}\right)}
$$

for $t \in \mathbf{Z} e_{2}+\mathbf{Z} e_{3}+\mathbf{Z} e_{4}$ and obtain

$$
\begin{aligned}
\alpha_{s}(t) & =\sum_{\omega \in \mathbf{Q} e_{2}+\mathbf{Q} e_{3}+Q e_{4} \bmod 1} \nu(\omega)^{-2 s} e^{2 \pi i \operatorname{Re}(\bar{\omega} t)} \\
& =\frac{1}{\zeta(I ; s)} \sum_{0 \neq c \in \Lambda} N(c)^{-s} \gamma(c, t),
\end{aligned}
$$

where $I=I^{(4)}$. Especially we have

$$
\alpha_{s}(0)=\frac{1}{\zeta(I ; s)} \sum_{0 \neq c \in \Lambda} \rho(c) N(c)^{1-s}=\frac{\zeta(I ; s-1) \zeta(2 s-3)}{\zeta(I ; s) \zeta(2 s-2)}
$$

Now let $t \neq 0$. A standard argument (cf. [6], 4.5) shows that

$$
\gamma(c, t)= \begin{cases}\rho(c) N(c) & \text { if } \operatorname{Re}\left(c^{-1} d \bar{t}\right) \in \mathbf{Z} \text { for all } d \in \mathscr{R}(c), \\ 0 & \text { otherwise. }\end{cases}
$$

Given $c=c_{2} c_{1}$, where $c_{1}, c_{2} \in \Lambda, N\left(c_{2}\right)=2^{m}, m \in \mathbf{N}_{0}, N\left(c_{1}\right)$ odd, we gain

$$
\gamma(c, t)=\gamma\left(c_{2}, t\right) \gamma\left(c_{1}, t\right)
$$

Using the isomorphism between $\Lambda / l \Lambda$ and $\operatorname{Mat}(2 ; Z / l Z)$ for odd $l \in \mathbf{N}$ (cf. [9], Vorlesung 8, resp. [17]) and a direct computation for $c_{2}$, one can show that $\operatorname{Re}\left(c^{-1} d \bar{t}\right) \in \mathbf{Z}$ holds for all $d \in \mathscr{R}(c)$ if and only if

$$
\rho(c) \mid \rho(t) \text { and } c t c^{-1} \in t+2 \rho(c) \operatorname{Alt}(1 ; \mathscr{O}) \text {. }
$$

Thus we calculate

$$
\begin{aligned}
\alpha_{s}(t) & =\frac{1}{\zeta(I ; s)} \sum_{l \in \mathrm{N}, l \mid \rho(t)} \sum_{t^{*} \in \operatorname{Alt}(1 ; \mathcal{O})} l^{3-2 s} \sum_{\substack{0 \neq c \in \Lambda, \rho(c)=1 \\
c \frac{1}{l} t=\left(\frac{1}{l} t+2 t^{*}\right) c}} N(c)^{1-s} \\
& =\frac{1}{\zeta(I ; s) \zeta(2 s-2)} \sum_{l \mid \rho(t)} l^{3-2 s} \sum_{t^{*} \in \operatorname{Alt}(1 ; \mathcal{O})} \sigma_{s-1}\left(t, t+2 l t^{*}\right) .
\end{aligned}
$$

Hence the assertion follows from Lemma 5.5.

Note that the sum over $t^{*}$ in the formula above is finite. 
In the case $\mathbf{F}=\mathbf{R}$ we are able to give the Fourier-expansions explicitly for $n=2,3$. Given $t \in \mathbf{N}$ and $s \in \mathbf{C}$ let

$$
\sigma_{s}(t):=\sum_{l \in \mathbf{N}, l \mid t} l^{s}
$$

denote the divisor sum. Then the application of Remark 2.3 and [19], p. 46 , resp. [20] leads to

Corollary 5.10. One has

$$
E_{2}^{\mathbf{R}}(Z, s)=\sum_{t \in \mathbf{Z}} c(Y ; t) e^{2 \pi i x t}, \quad Z=x J+Y \in \mathscr{H}(2 ; \mathbf{R})
$$

where

$$
\begin{aligned}
c(Y ; O)= & (\operatorname{det} Y)^{s}+(\operatorname{det} Y)^{s} \zeta_{1}(Y, 2 s) \\
& +\sqrt{\pi} \frac{\Gamma(2 s-1 / 2)}{\Gamma(2 s)} \cdot \frac{\zeta(4 s-1)}{\zeta(4 s)}(\operatorname{det} Y)^{1 / 2-s}, \\
c(Y ; t)= & 2 \pi^{2 s}|t|^{2 s-1 / 2} \frac{\sigma_{1-4 s}(|t|)}{\Gamma(2 s) \zeta(4 s)}(\operatorname{det} Y)^{1 / 4} K_{2 s-1 / 2}(2 \pi|t| \sqrt{\operatorname{det} Y})
\end{aligned}
$$

for $0 \neq t \in \mathbf{Z}$.

Note that the Fourier-coefficients $c(Y ; t)$ for $t \neq 0$ only depend on $\operatorname{det} Y$ and $s$.

Let $n \geq 3$ and fix a set of representatives $P: \operatorname{Pr}(n, 2 ; \mathbf{Z}) / \mathrm{GL}(2 ; \mathbf{Z})$. Then each $T \in \operatorname{Alt}^{\tau}(n ; Z)$ with rank $T=2$ possesses a unique representation

$$
T=\frac{1}{2} t J\left[P^{\prime}\right], \quad J=\left(\begin{array}{cc}
0 & 1 \\
-1 & 0
\end{array}\right),
$$

where $0 \neq t \in \mathbf{Z}$ and where $\varepsilon(2 T)=|t|$ is the greatest common divisor of the entries of $2 T \in \operatorname{Alt}(n ; \mathbf{Z})$. Now observe that

$$
t^{2} \cdot \operatorname{det}(Y[P])=2 \tau\left(T^{\prime} Y T, Y\right)
$$

holds. Hence we can combine the Corollaries 5.2 and 5.10 in order to gain

(5.7) $\quad E_{n, 2}^{\mathbf{R}}(Z, s)=\sqrt{\pi} \frac{\Gamma(2 s-1 / 2)}{\Gamma(2 s)} \frac{\zeta(4 s-1)}{\zeta(4 s)}(\operatorname{det} Y)^{s} \zeta_{2}\left(Y, 2 s-\frac{1}{2}\right)$

$$
\begin{aligned}
& +\sum_{\substack{T \in \operatorname{Alt}^{\tau}(n ; \mathbf{Z}) \\
\operatorname{rank} T=2}} 2 \pi^{2 s} \frac{\sigma_{4 s-1}(\varepsilon(2 T))}{\Gamma(2 s) \zeta(4 s)}(\operatorname{det} Y)^{s}\left(2 \tau\left(T^{\prime} Y T, Y\right)\right)^{\frac{1}{4}-s} \\
& \cdot K_{2 s-1 / 2}\left(2 \pi \sqrt{2 \tau\left(T^{\prime} Y T, Y\right)}\right) .
\end{aligned}
$$


Now let $n=3$. We compute

$$
\beta_{s}(Y ; 0)=(\operatorname{det} Y)^{1-2 s} \pi^{3 / 2} \frac{\Gamma(2 s-3 / 2)}{\Gamma(2 s)}
$$

in view of (5.6) and Lemma 4.8. Let $0 \neq T \in \operatorname{Alt}^{\tau}(3 ; Z)$ and $Y \in$ $\operatorname{Pos}(3 ; \mathbf{R})$. We choose $V \in \mathrm{GL}(3 ; \mathbf{R})$ such that $Y=V^{\prime} V$. Change of variables yields

$$
\begin{aligned}
& \beta_{S}(Y ; T)=\int_{\operatorname{Alt}(3 ; \mathbf{R})}(\operatorname{det}(Y+X))^{-2 s} e^{-2 \pi i \tau(X, T)} d X \\
& \quad=(\operatorname{det} Y)^{-2 s} \int_{\operatorname{Alt}(3 ; \mathbf{R})}\left(\operatorname{det}\left(I+X\left[V^{-1}\right]\right)\right)^{-2 s} e^{-2 \pi i \tau(X, T)} d X \\
& =(\operatorname{det} Y)^{1-2 s} \int_{\operatorname{Alt}(3 ; \mathbf{R})}(\operatorname{det}(I+X))^{-2 s} e^{-2 \pi i \tau\left(X, T\left[V^{\prime}\right]\right)} d X \\
& =(\operatorname{det} Y)^{1-2 s} \int_{-\infty}^{\infty} \int_{-\infty}^{\infty} \int_{-\infty}^{\infty}\left(1+x_{1}^{2}+x_{2}^{2}+x_{3}^{2}\right) e^{-2 \pi i \omega x_{1}} d x_{1} d x_{2} d x_{3}
\end{aligned}
$$

by the use of an orthogonal transformation, where

$$
\omega=\left(2 \tau\left(T\left[V^{\prime}\right], T\left[V^{\prime}\right]\right)^{1 / 2}=\left(2 \tau\left(T^{\prime} Y T, Y\right)\right)^{1 / 2} .\right.
$$

The same calculations as in the proof of Theorem 5.9 show that

$$
\begin{aligned}
\beta_{s}(Y ; T)= & 2 \pi^{2 s} \frac{1}{\Gamma(2 s)}\left(2 \tau\left(T^{\prime} Y T, Y\right)\right)^{s-3 / 4}(\operatorname{det} Y)^{1-2 s} \\
& \cdot K_{2 s-3 / 2}\left(2 \pi \sqrt{2 \tau\left(T^{\prime} Y T, Y\right)}\right)
\end{aligned}
$$

Given $0 \neq R \in \operatorname{Alt}(3 ; \mathbf{Q})$ note that $\nu(R)=l^{2}$, where $l \in \mathbf{N}$, if and only if $R=l^{-1} T$, where $T \in \operatorname{Alt}(3 ; \mathbf{Z})$ and $\varepsilon(T)=1$. Denoting the number of elements of a set $\mathscr{S}$ by $\# \mathscr{S}$, we calculate

$$
\begin{aligned}
\alpha_{s}(0) & =\sum_{R \bmod 1} \nu(R)^{-2 s} \\
& =\sum_{l=1}^{\infty} l^{-4 s} \cdot \#\left\{g \in \mathbf{Z}^{3} ; 1 \leq g_{j} \leq l \text {, g.c.d. } g=1\right\} \\
& =\frac{\zeta(4 s-3)}{\zeta(4 s)} .
\end{aligned}
$$

Given $0 \neq T \in \operatorname{Alt}^{\tau}(3 ; Z)$ we may restrict to the case

$$
T=\frac{1}{2}\left(\begin{array}{ccc}
0 & t & 0 \\
-t & 0 & 0 \\
0 & 0 & 0
\end{array}\right), \quad t=\varepsilon(2 T)
$$


in view of (5.5). Hence we calculate

$$
\begin{aligned}
\alpha_{s}(T) & =\sum_{R \bmod 1} \nu(R)^{-2 s} e^{2 \pi i \tau(R, T)} \\
& =\frac{1}{\zeta(4 s)} \sum_{l=1}^{\infty} \sum_{j=1}^{3} \sum_{q_{j}=1}^{l} l^{-4 s} e^{2 \pi i t q_{1} / l} \\
& =\frac{1}{\zeta(4 s)} \sigma_{3-4 s}(t) .
\end{aligned}
$$

A combination of (5.2), (5.3), (5.7) and Lemma 5.5 yields the final

COROLlaRY 5.11 .

$$
E_{3}^{\mathbf{R}}(Z, s)=\sum_{T \in \operatorname{Alt}^{\tau}(3 ; \mathbf{Z})} c(Y ; T) e^{2 \pi i \tau(X, T)}, \quad Z=X+Y \in \mathscr{H}(3 ; \mathbf{R}),
$$

where

$$
\begin{aligned}
& c(Y ; 0)=(\operatorname{det} Y)^{s}+(\operatorname{det} Y)^{s} \zeta_{1}(Y, 2 s) \\
& +\sqrt{\pi} \frac{\Gamma(2 s-1 / 2)}{\Gamma(2 s)} \frac{\zeta(4 s-1)}{\zeta(4 s)}(\operatorname{det} Y)^{s} \zeta_{2}(Y, 2 s-1 / 2) \\
& +\pi^{\frac{3}{2}} \frac{\Gamma(2 s-3 / 2)}{\Gamma(2 s)} \frac{\zeta(4 s-3)}{\zeta(4 s)}(\operatorname{det} Y)^{1-s} \text {, } \\
& c(Y ; T)=2 \pi^{2 s} \frac{\sigma_{4 s-1}(\varepsilon(2 T))}{\Gamma(2 s) \zeta(4 s)}(\operatorname{det} Y)^{s}\left(2 \tau\left(T^{\prime} Y T, Y\right)\right)^{1 / 4-s} \\
& \times K_{2 s-1 / 2}\left(2 \pi \sqrt{2 \tau\left(T^{\prime} Y T, Y\right)}\right) \\
& +2 \pi^{2 s} \frac{\sigma_{3-4 s}(\varepsilon(2 T))}{\Gamma(2 s) \zeta(4 s)}(\operatorname{det} Y)^{1-s}\left(2 \tau\left(T^{\prime} Y T, Y\right)\right)^{s-3 / 4} \\
& \times K_{2 s-3 / 2}\left(2 \pi \sqrt{2 \tau\left(T^{\prime} Y T, Y\right)}\right)
\end{aligned}
$$

for $T \neq 0$.

\section{REFERENCES}

[1] A. A. Albert, Structure of Algebras, Amer. Math. Soc. Publications, Providence 1961.

[2] H. Braun, Konvergenz verallgemeinerter Eisensteinscher Reihen, Math. Z., 44 (1939), 387-397.

[3] - Hermitian Modular Functions I, Ann. of Math., II. Ser., 50 (1949), 827-855. II, Ann. of Math., II. Ser., 51 (1950), 92-104. III, Ann. of Math., II. Ser., 53 (1951), 143-160.

[4] U. Christian, Siegelsche Modulfunktionen, 2. Auflage, Vorlesungsausarbeitung, Göttingen 1981. 
[5] L. E. Dickson, Algebras and their Arithmetics, Dover Publications, New York 1960.

[6] J. Elstrodt, F. Grunewald, J. Mennicke, Eisenstein series on three-dimensional hyperbolic space and imaginary quadratic number fields, J. Reine Angew. Math., 360 (1985), 160-213.

[7] E. Freitag, Siegelsche Modulfunktionen, Grundl. Math. Wiss. 254, SpringerVerlag, Berlin-Heidelberg-New York 1983.

[8] S. Helgason, Differential Geometry and Symmetric Spaces, Academic Press, New York-San Francisco-London 1962.

[9] A. Hurwitz, Vorlesungen über die Zahlentheorie der Quaternionen, SpringerVerlag, Berlin 1919.

[10] E. Kähler, Die Poincaré-Gruppe, Mathematica, Festschrift Ernst Mohr, 117144, Berlin 1985.

[11] Y. Kitaoka, Dirichlet series in the theory of Siegel modular forms, Nagoya Math. J., 95 (1984), 73-84.

[12] H. Klingen, Diskontinuierliche Gruppen in symmetrischen Räumen I, Math. Ann., 129 (1955), 345-369.

[13] M. Koecher,Über Dirichlet-Reihen mit Funktionalgleichung, J. Reine Angew. Math., 192 (1953), 1-23.

[14] An elementary approach to bounded symmetric domains, Lecture Notes, Rice 1969.

[15] - Lineare Algebra und analytische Geometrie, 2. Auflage, Grundwissen Math., 2, Springer-Verlag, Berlin-Heidelberg-New York-Tokyo 1985.

[16] A. Krieg, Modular Forms on Half-Spaces of Quaternions, Lecture Notes in Math. 1143, Springer-Verlag, Berlin-Heidelberg-New York-Tokyo 1985.

[17] - The elementary divisor theory over the Hurwitz order of integral quaternions, Linear Multilinear Algebra, 21 (1987), 325-344.

[18] L. Kronecker, Über bilineare Formen, J. Reine Angew. Math., 68 (1868), 273285 (= Werke I, 143-162).

[19] T. Kubota, Elementary Theory of Eisenstein Series, Kodansha, Tokyo 1973.

[20] H. Maßß, Automorphe Funktionen von mehreren Veränderlichen und Dirichletsche Reihen, Abh. Math. Sem. Univ. Hamburg, 16 (1949), 72-100.

[21] _ Die Differentialgleichungen in der Theorie der Siegelschen Modulfunktionen, Math. Ann., 126 (1953), 44-68.

[22] - Siegel's Modular Forms and Dirichlet Series, Lecture Notes in Math. 216, Springer-Verlag, Berlin-Heidelberg-New York 1971.

[23] _ Dirichletsche Reihen und Modulformen zweiten Grades, Acta Arith., 24 (1973), 225-238.

[24] W. Magnus, F. Oberhettinger and R. P. Soni, Formulas and theorems for special functions of mathematical physics, Grundl. Math. Wiss., 52, Springer-Verlag, New York 1966.

[25] M. Newman, Integral Matrices, Academic Press, New York-London 1972.

[26] G. Shimura, Confluent hypergeometric functions on tube domains, Math. Ann., 260 (1982), 269-302.

[27] _ On Eisenstein series, Duke Math. J., 50 (1983), 417-476.

[28] C. L. Siegel, Einheiten quadratischer Formen, Abh. Math. Sem. Hansische Univ., 13 (1940), 209-239 (= Ges. Abh.II, 138-168).

[29] _ Symplectic Geometry, Amer. J. Math., 65 (1943), 1-86 (= Ges. Abh.II, 274-359).

[30] , Die Modulgruppe in einer einfachen involutorischen Algebra, Festschrift Akad. Wiss. Göttingen 1951, 157-167 (= Ges. Abh.III, 143-153). 
[31] A. Terras, On automorphic forms for the general linear group, Rocky Mountain J. Math., 12 (1982), 123-143.

[32] _ Harmonic Analysis on Symmetric Spaces and Applications, II, to appear in Springer-Verlag.

[33] E. Witt, Eine Identität zwischen Modulformen zweiten Grades, Abh. Math. Sem. Hansische Univ., 14 (1941), 323-337.

[34] J. Wolf, Spaces of Constant Curvature, 4th edition, Publish or Perish, Berkeley 1977.

Received October 25, 1986 and in revised form July 8, 1987.

MATHEMATISCHES INSTITUT

EINSTEINSTRASSE 62

D-4400 MÜNSTER

Federal Republic of Germany 


\title{
PACIFIC JOURNAL OF MATHEMATICS
}

\section{EDITORS}

\author{
V. S. VARADARAJAN \\ (Managing Editor) \\ University of California \\ Los Angeles, CA 90024 \\ HERBERT ClEMENS \\ University of Utah \\ Salt Lake City, UT 84112 \\ R. FINN \\ Stanford University \\ Stanford, CA 94305
}

\author{
HERMANN FLASCHKA \\ University of Arizona \\ Tucson, AZ 85721
}

RAMESh A. GANGOLLI University of Washington Seattle, WA 98195

VAUGHAN F. R. JONES University of California

Berkeley, CA 94720

\author{
ROBION KIRBY \\ University of California \\ Berkeley, CA 94720 \\ C. C. MOORE \\ University of California \\ Berkeley, CA 94720 \\ HAROLD STARK \\ University of California, San Diego \\ La Jolla, CA 92093
}

\section{ASSOCIATE EDITORS}

\author{
R. ARENS \\ E. F. BECKENBACH \\ B. H. NEUMANN \\ F. WOLF \\ K. YOSHIDA \\ (1906-1982)

\section{SUPPORTING INSTITUTIONS}

\begin{abstract}
UNIVERSITY OF ARIZONA
UNIVERSITY OF BRITISH COLUMBIA

UNIVERSITY OF CALIFORNIA

MONTANA STATE UNIVERSITY

UNIVERSITY OF NEVADA, RENO

NEW MEXICO STATE UNIVERSITY

OREGON STATE UNIVERSITY
\end{abstract} \\ CALIFORNIA INSTITUTE OF TECHNOLOGY \\ UNIVERSITY OF OREGON \\ UNIVERSITY OF SOUTHERN CALIFORNIA \\ STANFORD UNIVERSITY \\ UNIVERSITY OF HAWAII \\ UNIVERSITY OF TOKYO \\ UNIVERSITY OF UTAH \\ WASHINGTON STATE UNIVERSITY \\ UNIVERSITY OF WASHINGTON
}

The Supporting Institutions listed above contribute to the cost of publication of this Journal, but they are not owners or publishers and have no responsibility for its content or policies.

Mathematical papers intended for publication in the Pacific Journal of Mathematics should be in typed form or offset-reproduced (not dittoed), double spaced with large margins. Please do not use built up fractions in the text of the manuscript. However, you may use them in the displayed equations. Underline Greek letters in red, German in green, and script in blue. The first paragraph must be capable of being used separately as a synopsis of the entire paper. In particular it should contain no bibliographic references. Please propose a heading for the odd numbered pages of less than 35 characters. Manuscripts, in triplicate, may be sent to any one of the editors. Please classify according to the scheme of Math. Reviews, Index to Vol. 39. Supply name and address of author to whom proofs should be sent. All other communications should be addressed to the managing editor, or Elaine Barth, University of California, Los Angeles, California 90024.

There are page-charges associated with articles appearing in the Pacific Journal of Mathematics. These charges are expected to be paid by the author's University, Government Agency or Company. If the author or authors do not have access to such Institutional support these charges are waived. Single authors will receive 50 free reprints; joint authors will receive a total of 100 free reprints. Additional copies may be obtained at cost in multiples of 50 .

The Pacific Journal of Mathematics is issued monthly as of January 1966. Regular subscription rate: $\$ 190.00$ a year (5 Vols., 10 issues). Special rate: $\$ 95.00$ a year to individual members of supporting institutions.

Subscriptions, orders for numbers issued in the last three calendar years, and changes of address should be sent to Pacific Journal of Mathematics, P.O. Box 969, Carmel Valley, CA 93924, U.S.A. Old back numbers obtainable from Kraus Periodicals Co., Route 100, Millwood, NY 10546.

The Pacific Journal of Mathematics at P.O. Box 969, Carmel Valley, CA 93924 (ISSN 0030-8730) publishes 5 volumes per year. Application to mail at Second-class postage rates is pending at Carmel Valley, California, and additional mailing offices. Postmaster: send address changes to Pacific Journal of Mathematics, P.O. Box 969, Carmel Valley, CA 93924.

\section{PUBLISHED BY PACIFIC JOURNAL OF MATHEMATICS, A NON-PROFIT CORPORATION}




\section{Pacific Journal of Mathematics}

\section{Vol. 133, No. 2 \\ April, 1988}

William Charles Bauldry, Attila Mate and Paul Nevai, Asymptotics for solutions of systems of smooth recurrence equations . . . . . . . . . . 209

Ehrhard Behrends, Isomorphic Banach-Stone theorems and isomorphisms which are close to isometries ............................229

Fernanda Maria Botelho, Rotation sets of maps of the annulus .........251

Edward Graham Evans, Jr. and Phillip Alan Griffith, Binomial behavior

of Betti numbers for modules of finite length . ................. 267

Andrei Iordan, Pseudoconvex domains with peak functions at each point of

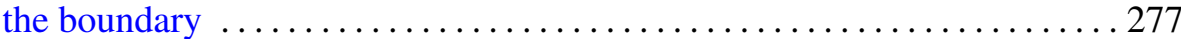

Zyun'iti Iwase, Dehn-surgery along a torus $T^{2}$-knot $\ldots \ldots \ldots \ldots \ldots \ldots . \ldots 289$

Marko Kranjc, Embedding 2-complexes in $\mathbf{R}^{4} \ldots \ldots \ldots \ldots \ldots \ldots \ldots \ldots \ldots$

Aloys Krieg, Eisenstein-series on real, complex, and quaternionic

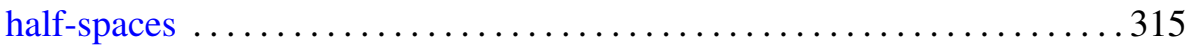

Masato Kuwata, Intersection homology of weighted projective spaces and

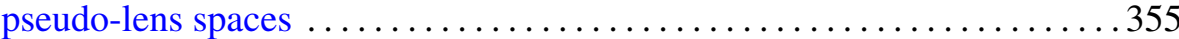

Carl Pomerance, András Sárközy and Cameron Leigh Stewart, On

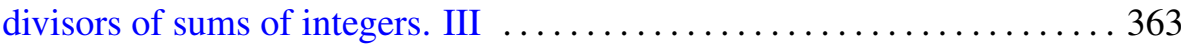

Martin Schechter, Potential estimates in Orlicz spaces ............... 381 